\title{
THE STRUCTURE OF MATRICES IN RATIONAL GAUSS QUADRATURE
}

\author{
CARL JAGELS AND LOTHAR REICHEL
}

\begin{abstract}
This paper is concerned with the approximation of matrix functionals defined by a large, sparse or structured, symmetric definite matrix. These functionals are Stieltjes integrals with a measure supported on a compact real interval. Rational Gauss quadrature rules that are designed to exactly integrate Laurent polynomials with a fixed pole in the vicinity of the support of the measure may yield better approximations of these functionals than standard Gauss quadrature rules with the same number of nodes. Therefore it can be attractive to approximate matrix functionals by these rational Gauss rules. We describe the structure of the matrices associated with these quadrature rules, derive remainder terms, and discuss computational aspects. Also discussed are rational Gauss-Radau rules and the applicability of pairs of rational Gauss and Gauss-Radau rules to computing lower and upper bounds for certain matrix functionals.
\end{abstract}

\section{INTRODUCTION}

Let $A \in \mathbb{R}^{n \times n}$ be a large, sparse or structured, symmetric, definite matrix, let $\boldsymbol{v} \in \mathbb{R}^{n}$ be a unit vector, and assume that the nonlinear function $f$ is defined and continuous on the closure of the spectrum of $A$. We are interested in computing approximations of matrix functionals of the form

$$
\mathcal{I}(f):=\boldsymbol{v}^{T} f(A) \boldsymbol{v}
$$

where the superscript ${ }^{T}$ denotes transposition. Functions $f$ that arise in applications include the exponential, the logarithm, and the square root; see, e.g., [1, 3, 5, 7, 10, 15, 19. We formulate our results for the case when $A$ is positive definite. The situation when $A$ is negative definite can be treated similarly; only minor modifications are required.

Introduce the spectral factorization

$$
A=Q \Lambda Q^{T}, \quad \Lambda=\operatorname{diag}\left[\lambda_{1}, \lambda_{2}, \ldots, \lambda_{n}\right], \quad Q^{T} Q=I,
$$

where $\lambda_{1} \leq \lambda_{2} \leq \ldots \leq \lambda_{n}$ are eigenvalues, the columns of $Q=\left[\boldsymbol{q}_{1}, \boldsymbol{q}_{2}, \ldots, \boldsymbol{q}_{n}\right] \in$ $\mathbb{R}^{n \times n}$ are orthonormal eigenvectors, and $I$ denotes the identity matrix. Substituting

Received by the editor August 15, 2011 and, in revised form, February 27, 2012.

2010 Mathematics Subject Classification. Primary 65F25, 65F60, 65D32.

Key words and phrases. Extended Krylov subspace, orthogonal Laurent polynomial, recursion relation, matrix functional, rational Gauss quadrature.

This research was supported in part by a grant from the Hanover College Faculty Development Committee.

This research was supported in part by NSF grant DMS-1115385. 
this factorization into (1.1) yields

$$
\mathcal{I}(f)=\sum_{j=1}^{n} f\left(\lambda_{j}\right) w_{j}^{2}, \quad w_{j}:=\boldsymbol{v}^{T} \boldsymbol{q}_{j} .
$$

This shows that (1.1) is a Stieltjes integral, which we may express as

$$
\mathcal{I}(f)=\int f(x) d \mu(x)
$$

where $\mu$ is an increasing piecewise constant distribution function with jumps at the eigenvalues $\lambda_{j}$ and $d \mu$ denotes the associated measure. Because of the connection between (1.1) and (1.3), we sometimes will refer to the function $f$ in (1.1) as the integrand.

Throughout this paper, we assume the matrix $A$ to be too large to allow the computation of its spectral factorization. Therefore, the representation (1.2) cannot be used for the evaluation of (1.1). The matrix $A$ is assumed to have a structure that makes the evaluation of matrix-vector products with $A$ and $A^{-1}$ feasible without forming the latter matrix. For instance, we may be able to use the Cholesky factorization of $A$, a fast Toeplitz solver [2, or a multilevel method to evaluate matrix-vector products with $A^{-1}$.

It is the purpose of this paper to discuss the structure of the matrices that arise when rational Gauss and Gauss-Radau quadrature rules are used to approximate functionals of the form (1.1). These rules are evaluated with the aid of a rational Lanczos process. Rational Gauss rules can be more attractive to use than standard Gauss rules for certain integrals; see below. We also describe how pairs of rational Gauss and Gauss-Radau rules or pairs of rational Gauss-Radau rules can be applied to determine upper and lower bounds for the functional (1.1) for certain integrands $f$. These bounds are analogous to bounds furnished by pairs of standard Gauss and Gauss-Radau rules or pairs of standard Gauss-Radau rules. Bounds based on standard Gauss and Gauss-Radau rules are described by Golub and Meurant [15, Chapter 7]; bounds involving certain rational Gauss-type quadrature rules also can be found in 23 .

We briefly review work by Golub and Meurant [14, or [15, Chapter 7] on the use of (standard) Gauss rules for the approximation of functionals (1.1). Application of $m$ steps of the (standard) Lanczos process to the matrix $A$ with initial vector $\boldsymbol{v}$ yields the decomposition

$$
A V_{m}=V_{m} T_{m}+\boldsymbol{g}_{m} \boldsymbol{e}_{m}^{T},
$$

where $V_{m}=\left[\boldsymbol{v}_{0}, \boldsymbol{v}_{1}, \ldots, \boldsymbol{v}_{m-1}\right] \in \mathbb{R}^{n \times m}$ has orthonormal columns with $\boldsymbol{v}_{0}=\boldsymbol{v}$, $T_{m} \in \mathbb{R}^{m \times m}$ is symmetric and tridiagonal with positive subdiagonal entries, and $\boldsymbol{g}_{m} \in \mathbb{R}^{n}$ satisfies $V_{m}^{T} \boldsymbol{g}_{m}=\mathbf{0}$. Here and throughout this paper $\boldsymbol{e}_{j}=[0, \ldots, 0,1$, $0, \ldots, 0]^{T}$ denotes the $j$ th axis vector of appropriate dimension. We are interested in the situation when $m \ll n$ and, in particular, assume $m$ to be small enough so that the decomposition (1.4) with the stated properties exists. Golub and Meurant [14] or [15, Chapter 7] show that

$$
\mathcal{G}_{m}(f):=\boldsymbol{e}_{1}^{T} f\left(T_{m}\right) \boldsymbol{e}_{1}
$$

is an $m$-point Gauss quadrature rule for the Stieltjes integral (1.3), i.e., $\mathcal{G}_{m}(p)=$ $\mathcal{I}(p)$ for all polynomials $p$ of degree at most $2 m-1$. The fact that $\mathcal{G}_{m}$ is an $m$ point quadrature rule can be seen by substituting the spectral factorization of $T_{m}$ 
into (1.5). Thus, the number of steps of the Lanczos process equals the number of nodes of the quadrature rule. To establish that $\mathcal{G}_{m}$ is a Gauss rule one first notices that the $j$ th column of $V_{m}$ can be expressed as $\boldsymbol{v}_{j-1}=p_{j-1}(A) \boldsymbol{v}$, where $p_{i-1}$ is an orthonormal polynomial of degree $j-1$ associated with the inner product 1

$$
(f, g):=(f(A) \boldsymbol{v})^{T} g(A) \boldsymbol{v}=\int f(x) g(x) d \mu(x) .
$$

We refer to [14, 15] for further details.

Gauss quadrature rules with few nodes yield accurate approximations of (1.3) when $f$ can be approximated well by a polynomial of low degree on the interval $\left[\lambda_{1}, \lambda_{n}\right]$. However, this is not the case in the situation when $f$ is analytic on $\left[\lambda_{1}, \lambda_{n}\right]$ and has a singularity nearby.

Example 1.1. Let the symmetric positive definite matrix $A$ have extreme eigenvalues $0<\lambda_{1}<\lambda_{n}$ with $\lambda_{1} / \lambda_{n}$ small. Then the accurate approximation of $f(t):=\ln (t)$ on $\left[\lambda_{1}, \lambda_{n}\right]$ by a polynomial requires the polynomial to be of high degree. Therefore the accurate approximation of $\boldsymbol{v}^{T} \ln (A) \boldsymbol{v}$ generally requires the use of a Gauss rule with many nodes. Consequently, many steps of the Lanczos process may be required to determine an approximation (1.5) of satisfactory accuracy. This is illustrated in Section 8 .

Druskin and Knizhnerman [9] showed that rational functions with a bounded single fixed pole may be able to approximate certain functions $f$, that are analytic on the interval $\left[\lambda_{1}, \lambda_{n}\right]$ and have a singularity nearby in the complex plane, more accurately than polynomials with the same number of coefficients. For this reason an $m$-point rational Gauss rule, which is designed to exactly integrate rational functions with a specified bounded pole, may yield a more accurate approximation of (1.3) than a standard Gauss rule (1.5) with the same number of nodes for this type of integrand; see also [4, 10, for error bounds for polynomial and rational approximation. The application of rational Gauss rules is particularly attractive when the matrix $A$ in the functional (1.1) has a structure that allows efficient evaluation of $A^{-1} \boldsymbol{w}$ for arbitrary vectors $\boldsymbol{w}$, e.g., by factorization or by application of an iterative method.

Rational Gauss quadrature rules were first investigated by Gonchar and López Lagomasino [17. These rules are Gauss quadrature rules for rational functions with prescribed poles. A more recent treatment is provided by Gautschi [12, Section 3.1.4]. We are interested in rational Gauss and Gauss-Radau rules for Laurent polynomials, which are rational functions whose only finite pole is at the origin, i.e., they are functions of the form $p(x) / x^{j}$, where $p$ is a polynomial of degree $k+j$. We show that the analog of the tridiagonal matrix $T_{m}$ associated with a (standard) $m$-point Gauss rule, for an $m$-point rational Gauss rule for Laurent polynomials is a pentadiagonal matrix $H_{m} \in \mathbb{R}^{m \times m}$ with a banded inverse. These properties of $H_{m}$ are used to establish the existence of rational Gauss rules for Laurent polynomials with arbitrary ratios larger than or equal to two of the numerator and denominator degrees. We also derive remainder terms for the rational Gauss and Gauss-Radau quadrature rules considered. The remainder terms show that for certain integrands $f$, pairs of rational Gauss and Gauss-Radau rules or pairs of suitable Gauss-Radau rules furnish upper and lower bounds for the functionals (1.1).

\footnotetext{
${ }^{1}$ Under suitable restrictions on the set of functions to which $f$ and $g$ belong, $(f, g)$ is an inner product; see Section 2 for further comments.
} 
This paper is organized as follows. Section 2 reviews available results on recursion formulas for orthonormal bases for rational Krylov subspaces associated with Laurent polynomials with a single real pole. These spaces sometimes are referred to as extended Krylov subspaces; see, e.g., 9]. The pentadiagonal structure of the matrix $H_{m}$ is discussed. This matrix is the orthogonal projection of $A$ onto a rational Krylov subspace. Let $G_{m} \in \mathbb{R}^{m \times m}$ denote the orthogonal projection of $A^{-1}$ onto this subspace. Section 3 shows that $G_{m}$ is banded, from which it follows that the inverse of $H_{m}$ has similar structure. Section 4 describes how the structure of $H_{m}$ and $G_{m}$ can be used to establish the existence of rational Gauss rules. The proofs are interesting because they use linear algebra techniques only. Remainder terms of a particular form are not required. It may be possible to apply the technique of proof to other classes of functions. However, since remainder terms are not used, the proof does not reveal the sign of the quadrature error. The sign is required to establish when rational Gauss and Gauss-Radau rules provide upper or lower bounds for the matrix functional (1.1). Therefore, we derive in Section [5 an expression for the quadrature error for rational Gauss rules. Comments on the computation of the nodes and weights of the rational Gauss rule determined by $H_{m}$ can be found in Section 6 . The computation of and the quadrature error for rational Gauss-Radau rules are described in Section 7 . These rules have one prescribed real node in the closed complement of the convex hull of the support of the measure $d \mu$. We show how upper and lower bounds for certain functionals (1.1) can be determined with the aid of pairs of rational Gauss-Radau rules. A few computed examples are presented in Section 8 and concluding remarks can be found in Section 9

\section{ReCURSion RELATiOns FOR EXtended Krylov SUbSPaCES}

This section reviews available results on recursion relations of orthonormal bases for rational Krylov subspaces of the form

$$
\mathbb{K}^{\ell, m}(A, \boldsymbol{v})=\operatorname{span}\left\{A^{-\ell+1} \boldsymbol{v}, \ldots, A^{-1} \boldsymbol{v}, \boldsymbol{v}, A \boldsymbol{v}, \ldots, A^{m-1} \boldsymbol{v}\right\} .
$$

Generically, $\mathbb{K}^{\ell, m}(A, \boldsymbol{v})$ is of dimension $m+\ell-1$, and $\mathbb{K}^{1, m}(A, \boldsymbol{v})=\mathbb{K}^{m}(A, \boldsymbol{v})$ is a standard Krylov subspace.

Njåstad and Thron [24] showed that orthonormal bases for the sequence of nested rational Krylov subspaces

$$
\mathbb{K}^{1,1}(A, \boldsymbol{v}) \subset \mathbb{K}^{2,2}(A, \boldsymbol{v}) \subset \ldots \subset \mathbb{K}^{m, m}(A, \boldsymbol{v}) \subset \ldots \subset \mathbb{R}^{n}
$$

satisfy a short recursion relation, i.e., the number of terms of the recursion relation is bounded independently of $m$. Their derivation uses properties of orthogonal Laurent polynomials. A survey of this and related results is provided by Jones and Njåstad 22].

An extension of the recursion relation discussed by Njåstad and Thron [24] to the sequence of rational Krylov subspaces

$$
\mathbb{K}^{1,2}(A, \boldsymbol{v}) \subset \mathbb{K}^{2,4}(A, \boldsymbol{v}) \subset \ldots \subset \mathbb{K}^{m, 2 m}(A, \boldsymbol{v}) \subset \cdots \subset \mathbb{R}^{n}
$$

is described in 20. More generally, sequences of nested rational Krylov subspaces of the form

$$
\mathbb{K}^{1, i+1}(A, \boldsymbol{v}) \subset \mathbb{K}^{2,2 i+1}(A, \boldsymbol{v}) \subset \ldots \subset \mathbb{K}^{m, m i+1}(A, \boldsymbol{v}) \subset \ldots \subset \mathbb{R}^{n},
$$


where $i$ is a positive integer are considered in [21. An extension in which the relation between the numerator and denominator degrees is more general than in (2.2) has recently been discussed by Díaz-Mendoza et al. 8.

Generation of an orthonormal basis for the subspace $\mathbb{K}^{m, m}(A, \boldsymbol{v})$ requires the evaluation of $m-1$ matrix-vector products with the matrix $A$ and the solution of $m-1$ linear systems of equations with $A$. For many matrices $A$, the evaluation of matrix-vector products can be carried out faster on modern computers than the solution of systems of equations with $A$, also when $A$ already is available in factored form. Therefore, typically an orthonormal basis for a subspace of the form (2.2) with $i>1$ can be computed faster than a basis for a space (2.1) of the same dimension. Computed examples in Section 8 show the rate of convergence of the computed approximation of (1.1) to be fairly insensitive to the choice of $i \geq 1$. It therefore can be attractive to use a value of $i$ larger than unity.

Recursion formulas for orthonormal bases for the nested spaces (2.2) can be derived by using the connection of the basis vectors with orthogonal Laurent polynomials. Introduce the spaces of Laurent polynomials

$$
\mathcal{L}_{j, k}:=\operatorname{span}\left\{x^{-j}, x^{-j+1}, \ldots, 1, \ldots, x^{k-1}, x^{k}\right\}, \quad j, k=0,1,2, \ldots,
$$

equipped with the inner product (1.6). Here and below, we assume that the dimension of the spaces (2.3) considered is small enough so that (1.6) indeed is an inner product.

Let $i$ be the integer in (2.2) and introduce the monic orthogonal Laurent polynomials

$$
\phi_{s}(x):= \begin{cases}x^{s}+\sum_{\ell=-\lfloor(s-1) / i\rfloor}^{s-1} c_{s, \ell} x^{\ell}, & s=1,2,3, \ldots, \\ x^{s}+\sum_{\ell=s+1}^{-i s} c_{s, \ell} x^{\ell}, & s=-1,-2,-3, \ldots,\end{cases}
$$

with $\phi_{0}(x):=1$. Here $\lfloor\alpha\rfloor$ denotes the largest integer smaller than or equal to $\alpha \in \mathbb{R}$. Then $\left\{\phi_{s}\right\}_{s=-m+1}^{i m}$ is a basis for $\mathcal{L}_{m-1, i m}$. We refer to the largest negative power of $\phi_{s}$ as the denominator degree and to the largest power of the numerator polynomial as the numerator degree. For instance, $\phi_{s}$ with $s \geq 1$ and non-vanishing trailing coefficient $c_{s,-\lfloor(s-1) / i\rfloor}$ has denominator degree $\lfloor(s-1) / i\rfloor$ and numerator degree $s+\lfloor(s-1) / i\rfloor$.

Let $j$ and $k$ be related according to $k=i j+\ell$, where $\ell$ is an integer such that $0 \leq \ell<i$. Then for $k>0$, we have

$$
\phi_{k} \perp \begin{cases}\mathcal{L}_{j, k-1} & \text { if } \ell \neq 0 \\ \mathcal{L}_{j-1, k-1} & \text { if } \ell=0 .\end{cases}
$$

Note that the vectors

$$
\boldsymbol{v}_{0}, \boldsymbol{v}_{1}, \ldots, \boldsymbol{v}_{i}, \boldsymbol{v}_{-1}, \boldsymbol{v}_{i+1}, \ldots, \boldsymbol{v}_{2 i}, \ldots, \boldsymbol{v}_{-m+1}, \ldots, \boldsymbol{v}_{i m}
$$

with $\boldsymbol{v}_{0}=\boldsymbol{v}$ constitute an orthonormal basis for the Krylov subspace $\mathbb{K}^{m, i m+1}(A, \boldsymbol{v})$. This basis can be expressed with the orthogonal Laurent polynomials (2.4); $\boldsymbol{v}_{j}$ is a multiple of $\phi_{j}(A) \boldsymbol{v}$. Hence, the determination of an orthonormal basis for $\mathbb{K}^{m, i m+1}(A)$ is equivalent to the generation of an orthonormal basis of Laurent polynomials for the space $\mathcal{L}_{m-1, i m}$. This connection is used in [21] to derive short recursion relations for the vectors (2.6). 
Suppose that the orthonormal basis (2.6) for $\mathbb{K}^{m, i m+1}(A, \boldsymbol{v})$ is available. Then the next set of $m+1$ orthonormal basis vectors $\boldsymbol{v}_{-m}, \boldsymbol{v}_{i m+1}, \boldsymbol{v}_{i m+2}, \ldots \boldsymbol{v}_{i(m+1)}$ can be generated recursively by

$$
\begin{aligned}
\delta_{-m} \boldsymbol{v}_{-m}= & \left(A^{-1}-\beta_{i m, i m} I\right) \boldsymbol{v}_{i m}-\beta_{i m, i m-1} \boldsymbol{v}_{i m-1} \\
& -\beta_{i m, i(m-1)+1} \boldsymbol{v}_{i(m-1)+1}-\beta_{i m,-m+1} \boldsymbol{v}_{-m+1},
\end{aligned}
$$

$$
\begin{aligned}
\delta_{i m+1} \boldsymbol{v}_{i m+1}= & \left(A-\alpha_{-m,-m} I\right) \boldsymbol{v}_{-m}-\alpha_{-m, i m} \boldsymbol{v}_{i m}, \\
\delta_{i m+2} \boldsymbol{v}_{i m+2}= & \left(A-\alpha_{i m+1, i m+1} I\right) \boldsymbol{v}_{i m+1} \\
& -\alpha_{i m+1,-m} \boldsymbol{v}_{-m}-\alpha_{i m+1, i m} \boldsymbol{v}_{i m}
\end{aligned}
$$

with $\alpha_{j, k}:=\boldsymbol{v}_{j}^{T} A \boldsymbol{v}_{k}$ and $\beta_{j, k}:=\boldsymbol{v}_{j}^{T} A^{-1} \boldsymbol{v}_{k}$. For $j=3,4, \ldots i$, we have the familiar three-term recursion formulas that arise in the standard Lanczos process,

$$
\begin{aligned}
\delta_{i m+j} \boldsymbol{v}_{i m+j}= & \left(A-\alpha_{i m+j-1, i m+j-1} I\right) \boldsymbol{v}_{i m+j-1} \\
& -\alpha_{i m+j-1, i m+j-2} \boldsymbol{v}_{i m+j-2} ;
\end{aligned}
$$

see 21] for details.

Define the matrix

$$
V_{m(i+1)}=\left[\boldsymbol{v}_{0}, \boldsymbol{v}_{1}, \ldots, \boldsymbol{v}_{i}, \boldsymbol{v}_{-1}, \boldsymbol{v}_{i+1}, \ldots, \boldsymbol{v}_{2 i}, \ldots, \boldsymbol{v}_{-m+1}, \ldots, \boldsymbol{v}_{i m}\right] \in \mathbb{R}^{n \times m(i+1)} .
$$

In order to simplify the subscripting, we henceforth will use the assignment

$$
\tau:=m(i+1) \text {. }
$$

The recursion coefficients in (2.7)-(2.10) determine a pentadiagonal matrix $H_{\tau}=$ $\left[h_{j, k}\right] \in \mathbb{R}^{\tau \times \tau}$ such that

$$
A V_{\tau}=V_{\tau} H_{\tau}+\boldsymbol{z}_{\tau} \boldsymbol{e}_{\tau}^{T}
$$

where

$$
\boldsymbol{z}_{\tau}=h_{\tau+1, \tau} \boldsymbol{v}_{-m}+h_{\tau+2, \tau} \boldsymbol{v}_{i m+1}
$$

Thus,

$$
H_{\tau}=V_{\tau}^{T} A V_{\tau}
$$

is the orthogonal projection of $A$ onto $\mathbb{K}^{m, i m+1}(A, \boldsymbol{v})$. Since $A$ is symmetric and definite, so is $H_{\tau}$.

Example 2.1. Consider the matrix $H_{\tau}$ for $i=3$ and $m=3$. Then $\tau=12$. The matrix $H_{12}$ may have non-vanishing entries in the positions marked by " $*$ ":

$$
H_{12}=\left[\begin{array}{cccccccccccc}
* & * & & & & & & & & & & \\
* & * & * & & & & & & & & & \\
& * & * & * & & & & & & & & \\
& & * & * & * & * & & & & & & \\
& & & * & * & * & & & & & & \\
& & & * & * & * & * & & & & & \\
& & & & & * & * & * & & & & \\
& & & & & * & * & * & * & & \\
& & & & & & * & * & * & & \\
& & & & & & * & * & * & * & \\
& & & & & & & & * & * & * \\
& & & & & & & & & * & *
\end{array}\right] .
$$


Example 2.2. The Laurent polynomials $\phi_{0}, \phi_{1}, \ldots, \phi_{i}$ are orthogonal polynomials with respect to the inner product (1.6) . Therefore the recursion formulas reduce to those of the Lanczos process (1.4) for the vectors $\boldsymbol{v}_{0}, \boldsymbol{v}_{1}, \ldots, \boldsymbol{v}_{i}$. It follows that the leading $(i+1) \times(i+1)$ principal submatrix of $H_{\tau}$, with $\tau$ given by (2.11), is tridiagonal.

Example 2.3. As usual, let $\tau$ be defined by (2.11). We describe the entries of the trailing $i \times i$ principal submatrix of $H_{\tau+i}$. This example will be used in the following section. The formulas (2.8), (2.9), and (2.10) for $j=3,4, \ldots, i$ can be expressed as

$$
\begin{gathered}
A\left[\boldsymbol{v}_{-m}, \boldsymbol{v}_{i m+1}, \boldsymbol{v}_{i m+2}, \ldots, \boldsymbol{v}_{i(m+1)-1}\right]=\left[\boldsymbol{v}_{-m}, \boldsymbol{v}_{i m+1}, \boldsymbol{v}_{i m+2}, \ldots, \boldsymbol{v}_{i(m+1)-1}\right] \hat{C} \\
+\alpha_{-m, i m} \boldsymbol{v}_{i m} \boldsymbol{e}_{1}^{T}+\alpha_{i m+1, i m} \boldsymbol{v}_{i m} \boldsymbol{e}_{2}^{T}+\delta_{i(m+1)} \boldsymbol{v}_{i(m+1)} \boldsymbol{e}_{i}^{T}
\end{gathered}
$$

where $\hat{C} \in \mathbb{R}^{i \times i}$ is given by

$$
\hat{C}=\left[\begin{array}{ccccc}
\alpha_{-m,-m} & \alpha_{i m+1,-m} & & & \mathrm{O} \\
\delta_{i m+1} & \alpha_{i m+1, i m+1} & \alpha_{i m+2, i m+1} & & \\
& \delta_{i m+2} & \alpha_{i m+2, i m+2} & \ddots & \\
& & \ddots & \ddots & \alpha_{i(m+1)-1, i(m+1)-2} \\
\mathrm{O} & & & \delta_{i(m+1)-1} & \alpha_{i(m+1)-1, i(m+1)-1}
\end{array}\right] .
$$

Thus, $\hat{C}$ is tridiagonal. It follows from

$$
\hat{C}=\left[\boldsymbol{v}_{-m}, \boldsymbol{v}_{i m+1}, \boldsymbol{v}_{i m+2}, \ldots, \boldsymbol{v}_{i(m+1)-1}\right]^{T} A\left[\boldsymbol{v}_{-m}, \boldsymbol{v}_{i m+1}, \boldsymbol{v}_{i m+2}, \ldots, \boldsymbol{v}_{i(m+1)-1}\right]
$$

that $\hat{C}$ is symmetric and definite. Thus,

$$
\alpha_{i m+1,-m}=\delta_{i m+1}, \quad \alpha_{i m+j, i m+j-1}=\delta_{i m+j}, \quad j=2,3, \ldots, i-1 .
$$

We show in Section 3 that the inverse of $H_{\tau}$ is banded. Moreover, the projection of $A^{-1}$ onto $\mathbb{K}^{m, i m+1}(A, \boldsymbol{v})$, given by

$$
G_{\tau}=V_{\tau}^{T} A^{-1} V_{\tau},
$$

is shown to be a rank-one modification of $H_{\tau}^{-1}$ and banded. The latter property is used in our discussion of rational Gauss quadrature in Section 4 . We refer to $G_{\tau}$ as the inverse projection matrix.

\section{The INVERSE PROJECTION MATRIX}

We present formulas for the entries $g_{j, k}$ of the matrix $G_{\tau}$ defined by (2.14) with $\tau$ given by (2.11). Let $V_{\tau}^{[j: k]}$ denote the submatrix made up of columns $j$ through $k$ of the matrix $V_{\tau}$, and let $G_{\tau}^{[h: j, k: l]}$ be the submatrix of $G_{\tau}$ consisting of the intersection of rows $h$ through $j$ and columns $k$ through $l$. For some integer $1 \leq l \leq m$, set $j=l(i+1)-i$ and consider the submatrices

$$
\begin{aligned}
V_{\tau}^{[j: j+i]}= & {\left[\boldsymbol{v}_{-l+1}, \boldsymbol{v}_{i(l-1)+1}, \boldsymbol{v}_{i(l-1)+2}, \ldots, \boldsymbol{v}_{i l-1}, \boldsymbol{v}_{i l}\right] \in \mathbb{R}^{n \times(i+1)}, } \\
V_{\tau}^{[j-(i+1): j+(i+1)]}= & {\left[\boldsymbol{v}_{-l+2}, \boldsymbol{v}_{i(l-2)+1}, \boldsymbol{v}_{i(l-2)+2}, \ldots, \boldsymbol{v}_{i(l-1)}, \boldsymbol{v}_{-l+1},\right.} \\
& \left.\boldsymbol{v}_{i(l-1)+1}, \boldsymbol{v}_{i(l-1)+2}, \ldots, \boldsymbol{v}_{i l-1}, \boldsymbol{v}_{i l}, \boldsymbol{v}_{-l}\right] \in \mathbb{R}^{n \times(2 i+3)} .
\end{aligned}
$$


The representation (3.2) is valid for $l \geq 2$; when $l=1$ the matrix has initial column $\boldsymbol{v}_{0}$ and only $i+2$ columns. Henceforth, we will assume that $l \geq 2$; results for the case $l=1$ can be shown analogously.

Multiplying equations (2.8) -2.10) by $A^{-1}$ and replacing $m$ by $l$ yields the relation

$$
A^{-1} V_{\tau}^{[j: j+i]} C=V_{\tau}^{[j-(i+1): j+(i+1)]} \tilde{G},
$$

where the matrix $C \in \mathbb{R}^{(i+1) \times(i+1)}$ has the structure

$$
C=\left[\begin{array}{cc}
\tilde{C} & \mathbf{0} \\
\delta_{i l} \boldsymbol{e}_{i}^{T} & 1
\end{array}\right], \quad \boldsymbol{e}_{i}, \mathbf{0} \in \mathbb{R}^{i}
$$

with an $i \times i$ leading principal submatrix $\tilde{C}$ of the form (2.13). In particular, $\tilde{C}$ is symmetric, tridiagonal, and definite. Its diagonal elements are given by

$$
\alpha_{-l+1,-l+1}, \alpha_{i(l-1)+1, i(l-1)+1}, \ldots, \alpha_{i l-1, i l-1}
$$

and its subdiagonal entries are

$$
\delta_{i(l-1)+1}, \ldots, \delta_{i l-1} ;
$$

see Example 3.1 below for further details.

The entries of the matrix $\tilde{G} \in \mathbb{R}^{(2 i+3) \times(i+1)}$ in (3.3) are defined as follows. Introduce the row vector

$$
\boldsymbol{b}_{k}=\left[\beta_{i k,-k+1}, \beta_{i k, i(k-1)+1}, \ldots, \beta_{i k, i k}\right] .
$$

Then the columns of $\tilde{G}$ can be written as

$$
\begin{array}{ll}
\text { col 1: } & {\left[-\alpha_{-l+1, i(l-1)} \boldsymbol{b}_{l-1}, 1-\alpha_{-l+1, i(l-1)} \delta_{-l+1}, 0, \ldots, 0\right]^{T}} \\
\operatorname{col} 2: & {\left[-\alpha_{i(l-1)+1, i(l-1)} \boldsymbol{b}_{l-1},-\alpha_{i(l-1)+1, i(l-1)} \delta_{-l+1}, 1,0, \ldots, 0\right]^{T}} \\
\operatorname{col} 3: & \boldsymbol{e}_{i+4} \\
\vdots & \vdots \\
\operatorname{col} i: & \boldsymbol{e}_{2 i+1} \\
\operatorname{col} i+1: & {\left[0, \ldots, 0, \boldsymbol{b}_{l}, \delta_{-l}\right]^{T} .}
\end{array}
$$

Example 3.1. Let $i=3$ and $l=3$. Then $j=9$ and the matrices $V_{\tau}^{[j: j+i]}$ and $V_{\tau}^{[j-(i+1): j+(i+1)]}$ in (3.3) are given by

$$
V_{\tau}^{[9: 12]}=\left[\boldsymbol{v}_{-2}, \boldsymbol{v}_{7}, \boldsymbol{v}_{8}, \boldsymbol{v}_{9}\right], \quad V_{\tau}^{[5: 13]}=\left[\boldsymbol{v}_{-1}, \boldsymbol{v}_{4}, \boldsymbol{v}_{5}, \boldsymbol{v}_{6}, \boldsymbol{v}_{-2}, \boldsymbol{v}_{7}, \boldsymbol{v}_{8}, \boldsymbol{v}_{9}, \boldsymbol{v}_{-3}\right] .
$$

Multiplying equations (2.8), (2.9), and (2.10) by $A^{-1}$ yields, in order,

$$
\left\{\begin{aligned}
\alpha_{-2,-2} A^{-1} \boldsymbol{v}_{-2}+\delta_{7} A^{-1} \boldsymbol{v}_{7} & =\boldsymbol{v}_{-2}-\alpha_{-2,6} A^{-1} \boldsymbol{v}_{6}, \\
\alpha_{7,-2} A^{-1} \boldsymbol{v}_{-2}+\alpha_{7,7} A^{-1} \boldsymbol{v}_{7}+\delta_{8} A^{-1} \boldsymbol{v}_{8} & =\boldsymbol{v}_{7}-\alpha_{7,6} A^{-1} \boldsymbol{v}_{6}, \\
\alpha_{8,7} A^{-1} \boldsymbol{v}_{7}+\alpha_{8,8} A^{-1} \boldsymbol{v}_{8}+\delta_{9} A^{-1} \boldsymbol{v}_{9} & =\boldsymbol{v}_{8},
\end{aligned}\right.
$$

and from (2.7) we obtain

$$
A^{-1} \boldsymbol{v}_{9}=\beta_{9,-2} \boldsymbol{v}_{-2}+\beta_{9,7} \boldsymbol{v}_{7}+\beta_{9,8} \boldsymbol{v}_{8}+\beta_{9,9} \boldsymbol{v}_{9}+\delta_{-3} \boldsymbol{v}_{-3} .
$$


Equations (3.5) and (3.6) can be expressed in the following form, after elimination of the terms $A^{-1} \boldsymbol{v}_{6}$ with the aid of (2.7),

$$
A^{-1} V_{\tau}^{[9: 12]} C=V_{\tau}^{[5: 13]}\left[\begin{array}{cccc}
* & * & & \\
* & * & & \\
* & * & & \\
* & * & & \\
* & * & & * \\
& * & & * \\
& & * & * \\
& & & * \\
& & &
\end{array}\right],
$$

where

$$
C=\left[\begin{array}{cccc}
\alpha_{-2,-2} & \alpha_{7,-2} & 0 & 0 \\
\delta_{7} & \alpha_{7,7} & \alpha_{8,7} & 0 \\
0 & \delta_{8} & \alpha_{8,8} & 0 \\
0 & 0 & \delta_{9} & 1
\end{array}\right]
$$

The leading $3 \times 3$ principal submatrix of $C$ is of the same form as the matrix (2.13) for $i=3$ and $m=2$. Thus, this leading principal submatrix is symmetric and definite. The matrix on the right-hand side of (3.7) corresponds to the matrix $\tilde{G}$ in (3.3). Entries that may be non-vanishing are marked by "*".

Since the submatrix $\tilde{C}$ is invertible, so is the matrix $C$ in (3.4). Post-multiplying equation (3.3) by $C^{-1}$ yields

$$
\begin{aligned}
A^{-1} V_{\tau}^{[j: j+i]} & =V_{\tau}^{[j-(i+1): j+(i+1)]} \tilde{G} C^{-1} \\
& =V_{\tau}^{[j-(i+1): j+(i+1)]} G^{[j-(i+1): j+(i+1), j: j+i]} .
\end{aligned}
$$

The matrix $G^{[j-(i+1): j+(i+1), j: j+i]}$ represents the non-vanishing entries of the columns $j$ through $j+i$ of $G_{\tau}$; these columns are associated with the block of vectors (3.1). We consider $j=l(i+1)-i$ for $2 \leq l \leq m$. The case $l=1$ can be treated similarly. Column $j$ of $G_{\tau}$ consists of the coefficients that express $A^{-1} \boldsymbol{v}_{-l+1}$ as a linear combination of the $2 i+3$ columns of $V_{\tau}^{[j-(i+1): j+(i+1)]}$. These coefficients generally are non-zero. Thus, column $j=l(i+1)-i$ of $G_{\tau}$ has at most $2 i+3$ non-vanishing entries, with $i+1$ of these entries occurring both above and below the diagonal entry.

We turn to column $j+1$ of $G_{\tau}$, and obtain from (3.2) and (3.8) that

$$
\begin{aligned}
A^{-1} \boldsymbol{v}_{i(l-1)+1}= & g_{j-(i+1), j+1} \boldsymbol{v}_{-l+2}+\sum_{k=j-i}^{j-1} g_{k, j+1} \boldsymbol{v}_{i(l-1)+k-j+1} \\
& +g_{j, j+1} \boldsymbol{v}_{-l+1}+\sum_{k=j+1}^{j+i} g_{k, j+1} \boldsymbol{v}_{i(l-1)+k-j} \\
& +g_{j+(i+1), j+1} \boldsymbol{v}_{-l} .
\end{aligned}
$$

The orthogonality of the vectors $\boldsymbol{v}_{j}$ and their relation to the Laurent polynomials $\phi_{k}$ give

$g_{j-(i+1), j+1}=\boldsymbol{v}_{-l+2}^{T} A^{-1} \boldsymbol{v}_{i(l-1)+1}=\left(\phi_{-l+2}, x^{-1} \phi_{i(l-1)+1}\right)=\left(\phi_{i(l-1)+1}, x^{-1} \phi_{-l+2}\right)$.

It follows from (2.5) that $\phi_{i(l-1)+1}$ is orthogonal to $\mathcal{L}_{(l-1), i(l-1)}$. Since $x^{-1} \phi_{i(l-1)+1}$ $\in \mathcal{L}_{(l-1), i(l-1)}$, we have $g_{j-(i+1), j+1}=0$. Similarly,

$$
\begin{aligned}
g_{j-i, j+1} & =\boldsymbol{v}_{l(i-2)+1}^{T} A^{-1} \boldsymbol{v}_{i(l-1)+1}=\left(\phi_{l(i-2)+1}, x^{-1} \phi_{i(l-1)+1}\right) \\
& =\left(\phi_{i(l-1)+1}, x^{-1} \phi_{l(i-2)+1}\right) .
\end{aligned}
$$


It follows from $x^{-1} \phi_{l(i-2)+1} \in \mathcal{L}_{(l-1), i(l-1)}$ that $g_{j-i, j+1}=0$. We can show similarly that all the first $i+1$ entries $g_{j-(i+1), j+1}, g_{j-i, j+1}, \ldots, g_{j-1, j+1}$ of the second column of $G_{\tau}$ vanish. The remaining columns follow the same pattern as the second one. Allowing $l$ to range from 1 to $m$, with $j=l(i+1)-i$, and using the symmetry shows the zero-structure of $G_{\tau}$. This establishes the following result.

Theorem 3.1. The symmetric matrix $G_{\tau}$ has bandwidth $i+1$. Its non-vanishing entries form $(i+2) \times(i+2)$ blocks on the diagonal, where the last diagonal entry of one block overlaps with the first diagonal entry of the succeeding block.

Example 3.2. The matrix $G_{\tau}$ for $i=3, m=3$, and $\tau=12$ may have nonvanishing entries in the positions marked by "*":

$$
G_{12}=\left[\begin{array}{cccccccccccc}
* & * & * & * & * & & & & & & & \\
* & * & * & * & * & & & & & & & \\
* & * & * & * & * & & & & & & & \\
* & * & * & * & * & & & & & & & \\
* & * & * & * & * & * & * & * & * & & & \\
& & & & * & * & * & * & * & & & \\
& & & & * & * & * & * & * & & & \\
& & & & * & * & * & * & * & & & \\
& & & & & * & * & * & * & * & * & * \\
& & & & & & & & * & * & * & * \\
& & & & & & & & * & * & * & * \\
& & & & & & & & & & * & *
\end{array}\right] .
$$

It follows from (3.8) that

$$
A^{-1} V_{\tau}=V_{\tau} G_{\tau}+\boldsymbol{v}_{-m} \boldsymbol{w}_{\tau}^{T},
$$

where

$$
\boldsymbol{w}_{\tau}=\sum_{k=0}^{i} g_{\tau+1, \tau-k} \boldsymbol{e}_{\tau-k} .
$$

We are in a position to discuss the relation between the matrices $G_{\tau}$ and $H_{\tau}$. Equations (2.12) and (3.9) yield

$$
I=\left(A V_{\tau}\right)^{T}\left(A^{-1} V_{\tau}\right)=H_{\tau} G_{\tau}+\boldsymbol{e}_{\tau} \boldsymbol{z}_{\tau}^{T} \boldsymbol{v}_{-m} \boldsymbol{w}_{\tau}^{T}=H_{\tau} G_{\tau}+h_{\tau+1, \tau} \boldsymbol{e}_{\tau} \boldsymbol{w}_{\tau}^{T} .
$$

Thus, $H_{\tau} G_{\tau}$ is a rank-one modification of the identity. The rank-one matrix may have non-vanishing elements only in the last $i+1$ entries of the last row. Since both the matrices $H_{\tau}$ and $G_{\tau}$ are invertible, so is the matrix

$$
I-h_{\tau+1, \tau} \boldsymbol{e}_{\tau} \boldsymbol{w}_{\tau}^{T} .
$$

Its inverse is given by

$$
\left[\begin{array}{cc}
I & 0 \\
c^{T} & c^{-1}
\end{array}\right]
$$

where

$$
c:=1-h_{\tau+1, \tau} g_{\tau+1, \tau}
$$

and

$$
\boldsymbol{c}:=[0, \ldots, 0, \underbrace{g_{\tau+1, \tau-i}, \ldots, g_{\tau+1, \tau-1}}_{i \text { entries }}]^{T} \in \mathbb{R}^{\tau-1} .
$$


Equation (3.10) yields

$$
H_{\tau}^{-1}=G_{\tau}\left(I-h_{\tau+1, \tau} \boldsymbol{e}_{\tau} \boldsymbol{w}_{\tau}^{T}\right)^{-1} .
$$

Partitioning $G_{\tau}$ in the same manner as (3.11) shows that

$$
\begin{aligned}
H_{\tau}^{-1} & =\left[\begin{array}{cc}
\hat{G} & \boldsymbol{d} \\
\boldsymbol{d}^{T} & g_{\tau, \tau}
\end{array}\right]\left[\begin{array}{cc}
I & \mathbf{0} \\
\boldsymbol{c}^{T} & c^{-1}
\end{array}\right] \\
& =\left[\begin{array}{cc}
\hat{\boldsymbol{G}}+\boldsymbol{d} \boldsymbol{c}^{T} & c^{-1} \boldsymbol{d} \\
c^{-1} \boldsymbol{d} & g_{\tau, \tau} c^{-1}
\end{array}\right],
\end{aligned}
$$

where $\hat{G}$ is the $(\tau-1) \times(\tau-1)$ leading principal submatrix of $G_{\tau}$ and

$$
\boldsymbol{d}:=[0, \ldots, 0, \underbrace{g_{\tau, \tau-i}, \ldots, g_{\tau, \tau-1}}_{i \text { entries }}]^{T} .
$$

Since all but possibly the last $i$ entries of $\boldsymbol{c}$ and $\boldsymbol{d}$ are known to vanish, the elements of the matrix $\boldsymbol{d} \boldsymbol{c}^{T}$ are zero except maybe those in its trailing $i \times i$ submatrix. This fact along with (3.12) shows that $H_{\tau}^{-1}$ differs from $G_{\tau}$ only in its trailing $(i+1) \times(i+1)$ submatrix. This gives us the following result.

Theorem 3.2. The matrix $H_{\tau}^{-1}$ has the same zero-structure as $G_{\tau}$.

\section{Application to rational Gauss quadrature}

We are in a position to discuss rational Gauss quadrature rules for the approximation of integrals of the form (1.1). The case of $i=1$ in (2.2) has been considered in 21], where it is shown that

$$
\boldsymbol{v}^{T} f(A) \boldsymbol{v}=\boldsymbol{e}_{1}^{T} f\left(H_{2 m}\right) \boldsymbol{e}_{1} \quad \forall f \in \mathcal{L}_{2 m-2,2 m+1} .
$$

Substituting the spectral decomposition of $H_{2 m}$ into $\boldsymbol{e}_{1}^{T} f\left(H_{2 m}\right) \boldsymbol{e}_{1}$ shows that this expression can be considered a quadrature rule with $2 m$ nodes, with the eigenvalues of $H_{2 m}$ being the nodes. A classical approach to rational Gauss quadrature rules for $f \in \mathcal{L}_{2 m-2,2 m+1}$ that does not explicitly utilize the recursion matrix $H_{2 m}$ is found in [22], where they are referred to as "strong Gaussian quadrature rules". This section demonstrates that more general rational Gauss rules exist for $f \in \mathcal{L}_{2 m-2,2 m i+1}$. That is, we will establish that

$$
\boldsymbol{v}^{T} f(A) \boldsymbol{v}=\boldsymbol{e}_{1}^{T} f\left(H_{\tau}\right) \boldsymbol{e}_{1} \quad \forall f \in \mathcal{L}_{2 m-2,2 m i+1},
$$

where as usual $\tau$ is given by (2.11).

The quadrature rule $\boldsymbol{e}_{1}^{T} f\left(H_{\tau}\right) \boldsymbol{e}_{1}$ has $\tau$ nodes, the eigenvalues of $H_{\tau}$. We note that it may not be necessary to compute the spectrum of $H_{\tau}$ in order to evaluate the quadrature rule; knowing the Cholesky factorization of $H_{\tau}$ or $-H_{\tau}$ may suffice. Discussions of many techniques for evaluating matrix functions with matrices of small to moderate size can be found in 19 .

Lemma 4.1. Let $H_{\tau}, G_{\tau}$, and $V_{\tau}$ be the matrices in (2.12) and (3.9). Let $\boldsymbol{v}=$ $V_{\tau} \boldsymbol{e}_{1}$. Then

$$
\begin{aligned}
\boldsymbol{v}^{T} A^{j} \boldsymbol{v} & =\boldsymbol{e}_{1}^{T} H_{\tau}^{j} \boldsymbol{e}_{1}, & & j=0,1, \ldots, 2 i m+1, \\
\boldsymbol{v}^{T} A^{-j} \boldsymbol{v} & =\boldsymbol{e}_{1}^{T} G_{\tau}^{j} \boldsymbol{e}_{1}, & & j=0,1, \ldots, 2 m-1
\end{aligned}
$$


Proof. We first consider (4.3). The relation (2.12) and the structure of $H_{\tau}$, illustrated in Example 2.1, yield

$$
A^{j} \boldsymbol{v}=V_{\tau} H_{\tau}^{j} \boldsymbol{e}_{1}, \quad j=0,1, \ldots, i m .
$$

Moreover,

$$
A^{i m+1} \boldsymbol{v}=V_{\tau} H_{\tau}^{i m+1} \boldsymbol{e}_{1}+\boldsymbol{z}_{\tau} \boldsymbol{e}_{\tau}^{T} H_{\tau}^{i m} \boldsymbol{e}_{1}
$$

Combining these expressions and using that $V_{\tau}^{T} \boldsymbol{z}_{\tau}=\mathbf{0}$ shows (4.3).

The relation (3.9) and the structure of $G_{\tau}$, illustrated in Example 3.2, give

$$
A^{-j} \boldsymbol{v}=V_{\tau} G_{\tau}^{j} \boldsymbol{e}_{1}, \quad j=0,1, \ldots, m,
$$

from which (4.4) follows.

In order to show that (4.1) is a rational Gauss rule, it remains to relate the powers $G_{\tau}^{j}$ in (4.4) to the negative powers $H_{\tau}^{-j}$. This is done in the following lemma.

Lemma 4.2. Let the matrices $H_{\tau}$ and $G_{\tau}$ be defined by (2.12) and (3.9), respectively, and let the matrix $A$ be definite. Then

$$
\boldsymbol{e}_{1}^{T} G_{\tau}^{j} \boldsymbol{e}_{1}=\boldsymbol{e}_{1}^{T} H_{\tau}^{-j} \boldsymbol{e}_{1}, \quad j=0,1, \ldots, 2 m-2 .
$$

Proof. It follows from (3.10) that

$$
H_{\tau} G_{\tau} \boldsymbol{e}_{1}=\left(I+\boldsymbol{e}_{\tau} \boldsymbol{u}_{\tau}^{T}\right) \boldsymbol{e}_{1}=\boldsymbol{e}_{1},
$$

where we have used that only the last $i+1$ entries of $\boldsymbol{u}_{\tau}$ and the first $i+2$ entries of the vector $G_{\tau} e_{1}$ may be non-zero. The latter follows from the structure of $G_{\tau}$. Since $A$ is definite, so is $H_{\tau}=V_{\tau}^{T} A V_{\tau}$. Hence,

$$
G_{\tau} e_{1}=H_{\tau}^{-1} e_{1} \text {. }
$$

Further, if $m \geq 3$, then

$$
H_{\tau}^{2} G_{\tau}^{2} \boldsymbol{e}_{1}=H_{\tau}\left(I+\boldsymbol{e}_{\tau} \boldsymbol{u}_{\tau}^{T}\right) G_{\tau} \boldsymbol{e}_{1}=H_{\tau} G_{\tau} \boldsymbol{e}_{1}=\boldsymbol{e}_{1},
$$

where we have used that only the first $i+2$ entries of the vector $G_{\tau} \boldsymbol{e}_{1}$ may be non-zero. Proceeding similarly for increasing values of $j$ yields

$$
H_{\tau}^{j} G_{\tau}^{j} \boldsymbol{e}_{1}=\boldsymbol{e}_{1}, \quad j=0,1, \ldots, m-1 .
$$

This shows (4.5).

Theorem 4.3 (Rational Gauss quadrature). Equation (4.2) holds, i.e., the righthand side is a rational Gauss quadrature rule for the approximation of (1.1).

Proof. The result follows by combining (4.3)- (4.5).

We find the above proof of interest because it relies on linear algebra techniques only. Quadrature rules for other basis functions with associated matrices $H_{\tau}$ and $G_{\tau}$ that satisfy the properties (4.3)-(4.5) also may be considered Gauss rules. However, the proof does not provide an expression for the quadrature error. Such an expression is helpful for establishing for which integrands $f$ the quadrature rule provides an upper or lower bound for the matrix functional (1.1). The following section derives a formula for the quadrature error for rational Gauss rules of the type considered in this paper. 


\section{The error in Rational Gauss Rules}

This section presents an algebraic derivation of rational Gauss quadrature rules with the aim to determine an expression for the quadrature error. The following theorem is an analog of a well-known result for orthogonal polynomials. The conditions of the theorem are satisfied by the monic orthogonal Laurent polynomials defined by (2.4).

Theorem 5.1. Let $\psi \in \mathcal{L}_{j, k}$ be of exact denominator degree $j$ and of exact numerator degree $j+k$. Suppose further that $\psi$ is orthogonal to either $\mathcal{L}_{j, k-1}$ or $\mathcal{L}_{j-1, k}$ with respect to the inner product (1.6). Then the following holds:

i. $\psi$ has $j+k$ zeros.

ii. The zeros are real, distinct, and lie in the convex hull of the support of $d \mu$.

Proof. The conditions of exactness imply that

$$
\psi(x)=\frac{P(x)}{x^{j}}
$$

where $P$ is a polynomial of exact degree $k+j$. First assume that $\psi$ is orthogonal to $\mathcal{L}_{j, k-1}$. Then $P$ is orthogonal to polynomials of degree less than $k+j$ with respect to the measure $d \mu(x) / x^{j}$, and the proof of $i$ now follows from the standard proof of the analogous result for orthogonal polynomials with respect to a non-negative measure; see, for example, [12, p. 7]. Similarly, when $\psi$ is orthogonal to $\mathcal{L}_{j-1, k}$, it follows that $P$ is orthogonal to polynomials of degree less than $k+j$ with respect to the measure $d \mu(x) / x^{j-1}$. Again, property $i$ follows from the analogous result for orthogonal polynomials with respect to a non-negative measure.

Let the function $\psi$ satisfy the conditions of Theorem 5.1 with $\psi \perp \mathcal{L}_{j, k-1}$. Denote the $j+k$ distinct zeros of $\psi$ by

$$
x_{1}<x_{2}<\ldots<x_{j+k}
$$

and introduce the Laurent-Lagrange polynomials

They satisfy

$$
l_{\nu}(x):=\frac{\psi(x)}{\left(x-x_{\nu}\right) \psi^{\prime}\left(x_{\nu}\right)}, \quad \nu=1,2, \ldots, j+k .
$$

The Laurent polynomial

$$
l_{\nu}\left(x_{\iota}\right)=\left\{\begin{array}{lll}
1 & \text { if } \quad \iota=\nu \\
0 & \text { if } \quad \iota \neq \nu
\end{array}\right.
$$

$$
L(x):=\sum_{\nu=1}^{j+k} l_{\nu}(x) f\left(x_{\nu}\right)
$$

interpolates $f$ at the zeros of $\psi$. Suppose that $f \in \mathcal{L}_{2 j, 2 k-1}$. Then the $k+j$ zeros of $\psi(x)$ are shared by $f(x)-L(x)$. Hence, there is a Laurent polynomial $r \in \mathcal{L}_{j, k-1}$ such that

$$
f(x)-L(x)=\psi(x) r(x) .
$$

Integrating this relation with respect to the measure $d \mu$ (cf. (1.3)) yields

$$
\begin{aligned}
\mathcal{I}(f) & =\int L(x) d \mu(x)+\int \psi(x) r(x) d \mu(x) \\
& =\sum_{\nu=1}^{j+k} f\left(x_{\nu}\right) \int l_{\nu}(x) d \mu(x) .
\end{aligned}
$$


This is a quadrature rule with weights

$$
w_{\nu}=\int l_{\nu}(x) d \mu(x)
$$

whose positivity can be established by substituting the squared Laurent-Lagrange polynomial $l_{\nu}^{2}$ for $f$ in (5.1) to obtain

$$
\int l_{\nu}^{2}(x) d \mu(x)=w_{\nu}, \quad \nu=1,2, \ldots, j+k .
$$

If on the other hand $\psi \perp \mathcal{L}_{j-1, k}$, then we choose $f \in \mathcal{L}_{2 j-1,2 k}$ and $r \in \mathcal{L}_{j-1, k}$. These observations yield the following results.

Theorem 5.2. Let $x_{1}, x_{2}, \ldots, x_{j+k}$ be the distinct zeros of $\psi \in \mathcal{L}_{j, k}$. Consider the quadrature rule

$$
\mathcal{G}_{\kappa}(f)=\sum_{\nu=1}^{\kappa} w_{\nu} f\left(x_{\nu}\right), \quad \kappa=j+k,
$$

with positive weights $w_{\nu}$ defined by (5.2). Then the following results hold:

i. $\psi \perp \mathcal{L}_{j-1, k}$ if and only if the rule is exact for $f \in \mathcal{L}_{2 j-1,2 k}$.

ii. $\psi \perp \mathcal{L}_{j, k-1}$ if and only if the rule is exact for $f \in \mathcal{L}_{2 j, 2 k-1}$.

Proof. Consider case $i$. The fact that $\psi \perp \mathcal{L}_{j-1, k}$ implies that the quadrature rule is exact for $f \in \mathcal{L}_{2 j-1,2 k}$ follows from the discussion preceding the theorem. Conversely, suppose that the rule is exact for $f \in \mathcal{L}_{2 j-1,2 k}$. Then from the definition of the inner product (1.6), we obtain

$$
\left(x^{s}, \psi(x)\right)=\mathcal{G}_{\kappa}\left(x^{s} \psi(x)\right)=0, \quad s=-j+1,-j+2, \ldots, k .
$$

Thus, $\psi \perp \mathcal{L}_{j-1, k}$. This establishes property $i$. Property $i i$ can be shown in a similar manner.

The following result is a consequence of the above theorem.

Corollary 5.3. Let $\tau$ be defined by (2.11) and consider the sequence of orthogonal Laurent polynomials

$$
\phi_{0}, \phi_{1}, \ldots, \phi_{i}, \phi_{-1}, \phi_{i+1}, \ldots, \phi_{2 i}, \phi_{-2}, \phi_{2 i+1}, \ldots, \phi_{i m}, \phi_{-m}, \ldots .
$$

The rational Gauss rule $\mathcal{G}_{\tau}(f)$, whose nodes are the zeros of $\phi_{-m}$ in the sequence (5.3), is exact for all $f \in \mathcal{L}_{2 m-1,2 i m}$.

Moreover, consider the modified sequence

$$
\phi_{0}, \phi_{1}, \ldots, \phi_{i}, \phi_{-1}, \phi_{i+1}, \ldots, \phi_{2 i}, \phi_{-2}, \phi_{2 i+1}, \ldots, \phi_{i m}, \phi_{i m+1}, \ldots,
$$

and let the nodes of the rational Gauss rule $\mathcal{G}_{\tau}(f)$ be the zeros of $\phi_{i m+1}$. Then this rule is exact for all $f \in \mathcal{L}_{2 m-2,2 i m+1}$. This quadrature rule is the same as (4.2).

Proof. The property of the rational Gauss rule based on the sequence (5.3) follows from property $i$ of Theorem 5.2 , the statement about the rational Gauss rule based on the sequence (5.4) is a consequence of property ii of the theorem.

An expression for the quadrature error $\left(\mathcal{I}-\mathcal{G}_{\kappa}\right)(f)$ with $\kappa=j+k$ can be derived by considering the Laurent-Hermite interpolation polynomial

$$
\hat{L}(x):=\sum_{\nu=1}^{\kappa}\left(\hat{l}_{\nu}(x) f\left(x_{\nu}\right)+\tilde{l}_{\nu}(x) f^{\prime}\left(x_{\nu}\right)\right),
$$


where

$$
\begin{aligned}
& \hat{l}_{\nu}(x)=\left[1-2\left(x-x_{\nu}\right) l_{\nu}^{\prime}\left(x_{\nu}\right)\right] l_{\nu}^{2}(x), \\
& \tilde{l}_{\nu}(x)=\left(x-x_{\nu}\right) l_{\nu}^{2}(x) .
\end{aligned}
$$

Analogously to standard Hermite polynomial interpolation, we have

$$
\hat{L}\left(x_{\nu}\right)=f\left(x_{\nu}\right), \quad \hat{L}^{\prime}\left(x_{\nu}\right)=f^{\prime}\left(x_{\nu}\right), \quad \nu=1,2, \ldots, j+k .
$$

Theorem 5.4 (Laurent-Hermite interpolation error). Let $\hat{L}$ be the Laurent-Hermite polynomial (5.5) determined by the interpolation nodes $x_{1}<x_{2}<\ldots<x_{\kappa}$, the $\kappa=j+k$ zeros of $\psi \in \mathcal{L}_{j, k}$ with leading coefficient $d$. Assume that $f$ is $2 \kappa$ times continuously differentiable in the open interval between the nodes $x_{1}$ and $x_{\kappa}$. Then for some scalar $c=c(x)$ dependent on $x$ in this interval, we have

$$
f(x)=\hat{L}(x)+\frac{d^{2 \kappa}}{d t^{2 \kappa}}\left(t^{2 j} f(t)\right)_{t=c} \frac{\psi^{2}(x)}{d^{2}(2 \kappa) !} .
$$

Proof. If $x=x_{\nu}$ for some $\nu=1,2, \ldots, \kappa$, then (5.6) holds for an arbitrary constant $c$. Assume that $x \neq x_{\nu}$ for all $\nu$. We then observe that

$$
\psi(x)=\frac{P(x)}{x^{j}},
$$

where $P(x)$ is polynomial of degree $\kappa$, whose zeros are those of $\psi(x)$. Next note that $x^{2 j} \hat{L}(x)$ is a polynomial of degree $2 \kappa-1$ and consider

$$
g(t)=t^{2 j}(f(t)-\hat{L}(t))-x^{2 j}(f(x)-\hat{L}(x)) \frac{P^{2}(t)}{P^{2}(x)} .
$$

The function $g$ is $2 \kappa$ times continuously differentiable and $g\left(x_{\nu}\right)=g^{\prime}\left(x_{\nu}\right)=0$ for $\nu=1,2, \ldots, \kappa$. Further, for any fixed $x \neq x_{\nu}$ for all $\nu$, we have $g(x)=0$. Hence, $g$ has $2 \kappa+1$ zeros, counting multiplicities. By Rolle's theorem, there exists a scalar $c=c(x)$ depending on $x$ in the open interval between the nodes $x_{1}$ and $x_{\kappa}$, for which

$$
\begin{aligned}
0 & =\frac{d^{2 \kappa}}{d t^{2 \kappa}}(g(t))_{t=c} \\
& =\frac{d^{2 \kappa}}{d t^{2 \kappa}}\left(t^{2 j} f(t)\right)_{t=c}-x^{2 j}(f(x)-\hat{L}(x)) \frac{d^{2 \kappa}}{d t^{2 \kappa}}\left(\frac{P^{2}(t)}{P^{2}(x)}\right)_{t=c}
\end{aligned}
$$

Noting that $P^{2}(t)$ is a polynomial of degree $2 \kappa$ with leading coefficient $d^{2}$, rearranging terms, and dividing by $x^{2 j}$ yields (5.6).

The following result is an application of Theorem 5.4

Corollary 5.5. Let $\kappa=j+k$ and assume that $f$ is $2 \kappa$ times continuously differentiable in the convex hull of the support of the measure $d \mu$. The quadrature rule $\mathcal{G}_{\kappa}$ satisfy condition ii of Theorem [5.2, Then

$$
\left(\mathcal{I}-\mathcal{G}_{\kappa}\right)(f)=\frac{d^{2 \kappa}}{d t^{2 \kappa}}\left(t^{2 j} f(t)\right)_{t=c} \frac{\mathcal{I}\left(\psi^{2}\right)}{d^{2}(2 \kappa) !}
$$

for some scalar $c$ in the convex hull of the support of the measure $d \mu$, where $\psi$ is defined in Theorem 5.2 . 
Proof. Since the quadrature rule $\mathcal{G}_{\kappa}$ satisfies condition $i i$ of Theorem 5.2, it is exact for functions in $\mathcal{L}_{2 j, 2 k-1}$. The Laurent-Hermite interpolation polynomial $\hat{L}$ lives in $\mathcal{L}_{2 j, 2 k-1}$. Therefore, $\mathcal{I}(\hat{L})=\mathcal{G}_{\kappa}(\hat{L})$, and we obtain

$$
\left(\mathcal{I}-\mathcal{G}_{\kappa}\right)(f)=\mathcal{I}(f-\hat{L})=\mathcal{I}\left(\frac{d^{2 \kappa}}{d t^{2 \kappa}}\left(t^{2 j} f(t)\right)_{t=c(x)} \frac{\psi^{2}(x)}{d^{2}(2 \kappa) !}\right),
$$

where the last equality follows from Theorem [5.4 integration is carried out with respect to the variable $x$. The corollary now follows from an application of the mean-value theorem of integration.

We will now apply the above results to determine an expression for the error for the quadrature rule (4.2). Introduce the orthonormal Laurent polynomials

$$
\tilde{\phi}_{s}(x):=\frac{\phi_{s}(x)}{\left\|\phi_{s}\right\|}, \quad s=0,1, \ldots, i,-1, i+1, i+2, \ldots,
$$

and the vector

$$
\Phi_{\tau}(x):=\left[\tilde{\phi}_{0}(x), \ldots, \tilde{\phi}_{i}(x), \tilde{\phi}_{-1}(x), \ldots, \tilde{\phi}_{-m+1}(x), \ldots, \tilde{\phi}_{i m}(x)\right]^{T},
$$

with $\tau$ defined by (2.11). The vector form of the matrix formula (2.12) is given by

$$
x \Phi_{\tau}(x)=H_{\tau} \Phi_{\tau}(x)+\left(h_{\tau+1, \tau} \tilde{\phi}_{-m}(x)+h_{\tau+2, \tau} \tilde{\phi}_{i m+1}(x)\right) \boldsymbol{e}_{\tau} .
$$

Observe that the zeros of the Laurent polynomial

$$
\psi_{\tau}(x)=h_{\tau+1, \tau} \tilde{\phi}_{-m}(x)+h_{\tau+2, \tau} \tilde{\phi}_{i m+1}(x)
$$

are the eigenvalues $\left\{\theta_{i}\right\}_{i=1}^{\tau}$ of $H_{\tau}$. Further, $\tilde{\phi}_{-m}$ and $\tilde{\phi}_{i m+1}$ have exact denominator degree $m$ and exact numerator degrees $\tau$ and $\tau+1$, respectively. Since $\psi_{\tau}$ only has $\tau$ zeros, the constant term of the numerator of the right-hand side of (5.9) must vanish. It follows that $\psi_{\tau}(x)$ is a multiple of

$$
\frac{\Pi_{s=1}^{\tau}\left(x-\theta_{s}\right)}{x^{m-1}} .
$$

Hence, $\psi_{\tau} \in \mathcal{L}_{m-1, i m+1}$. Since the quadrature rule $\mathcal{G}_{\tau}(f)$ defined by (4.2) is exact for $f \in \mathcal{L}_{2 m-2,2 i m+1}$, we obtain from Theorem 5.2 that $\psi_{\tau} \perp \mathcal{L}_{m-1, i m}$. Thus, condition $i$ of Theorem 5.1 is satisfied with $j=m-1$ and $k=i m+1$, and it follows that the expression for the quadrature error $\left(\mathcal{I}-\mathcal{G}_{\kappa}\right)(f)$ of Corollary 5.5 holds with $\kappa=\tau$ and $j=m-1$ for functions $f$ that satisfy the conditions of the corollary.

Corollary 5.6. Let the open interval $(a, b)$ contain the spectrum of $A$ and let the function $f$ be $2 \tau$ times continuously differentiable in this interval. Assume that

$$
\frac{d^{2 \tau}}{d x^{2 \tau}}\left(x^{2(m-1)} f(x)\right) \geq 0, \quad a<x<b .
$$

Then

$$
\mathcal{G}_{\tau}(f) \leq \mathcal{I}(f)
$$

or, equivalently,

$$
\boldsymbol{e}_{1}^{T} f\left(H_{\tau}\right) \boldsymbol{e}_{1} \leq \boldsymbol{v}^{T} f(A) \boldsymbol{v}
$$

Proof. The inequality follows from Corollary 5.5 with $\kappa=\tau$ and $j=m-1$. 


\section{Computation of nodes and weights of rational Gauss rules}

The eigenvalues and squared first component of associated normalized eigenvectors of the matrix $H_{\tau}$ in (4.2) with $\tau$ given by (2.11) yield the nodes and weights, respectively, of the $\tau$-point rational Gauss quadrature rule determined by $H_{\tau}$. The nodes and weights can be computed in several ways. For instance, we may first bring $H_{\tau}$ to tridiagonal form by orthogonal similarity transformation using a suitably chosen sequence of Givens rotations and then apply the Golub-Welsch algorithm 16. Alternatively, we may use a divide-and-conquer technique to split $H_{\tau}$ into, say $m$, smaller matrices $H_{\tau}^{(\ell)}, \ell=1,2, \ldots, m$, with symmetric rank-one modifications and determine the eigenvalues, and first and last components of the normalized eigenvectors of each one of the smaller matrices $H_{\tau}^{(\ell)}$; see [6, 18, for discussions on divide-and-conquer methods for symmetric tridiagonal matrices. A comparison of these schemes will be presented elsewhere.

An alternative approach to determine the rational Gauss rule in (4.2), described, e.g., by Gautschi [12, Theorem 3.25], is to first compute the symmetric tridiagonal matrix $T_{\tau} \in \mathbb{R}^{\tau \times \tau}$ associated with the measure $d \mu$ in (1.3) and defined by (1.2). This matrix can be determined by carrying out $\tau$ steps of the Lanczos process applied to $A$ with initial vector $\boldsymbol{v}$; cf. (1.4). The matrix $T_{\tau}$ then is modified to yield a new symmetric tridiagonal matrix $\breve{T}_{\tau}$ associated with the measure

$$
d \breve{\mu}(x):=\frac{d \mu(x)}{x^{2 m-2}} .
$$

The entries of the matrix $\breve{T}_{\tau}$ are recursion coefficients for orthonormal polynomials associated with the measure (6.1). Efficient algorithms for computing $\breve{T}_{\tau}$ from $T_{\tau}$ are available; see Gautschi [12, Section 2.4] for a discussion and references. This method for determining rational Gauss rules works well when $m$ is small, say, $m \leq 2$. However, for large values of $m$, numerical instability may significantly reduce the accuracy of the computed approximation of $\breve{T}_{\tau}$. Further details and computed illustrations for small $m$ are presented in [23].

\section{Gauss-Radau Rules}

The standard $(m+1)$-point Gauss-Radau rule for the integral (1.3) is a quadrature rule that is exact for all polynomials of degree at most $2 m$ and has a fixed node in the closure of the complement of the convex hull of the support of the measure $d \mu$; see, e.g., Gautschi [12]. This section discusses the computation of rational Gauss-Radau rules and error bounds that can be determined with these rules. In the remainder of this paper, we refer to rational Gauss-Radau quadrature rules briefly as rational Radau rules.

Standard $m$-point Gauss-Radau rules for the approximation of matrix functionals (1.1) can be determined by modifying the tridiagonal matrix $T_{m}$ in the decomposition (1.4), which can be evaluated by carrying out $m$ steps of the (standard) Lanczos process; see, e.g., [11, 12, 13] or [15, Section 6.2] for details. We show that rational Radau rules can be determined in an analogous fashion. Express the decomposition (5.8) in the form

$$
x \Phi_{\tau}(x)=H_{\tau} \Phi_{\tau}(x)+\psi_{\tau}(x) \boldsymbol{e}_{\tau},
$$

where the vector $\Phi_{\tau}(x) \in \mathbb{R}^{\tau}$ is defined by (5.7), the matrix $H_{\tau} \in \mathbb{R}^{\tau \times \tau}$ of recursion coefficients is pentadiagonal, $\tau$ is given by (2.11), and $\psi_{\tau}$ is a linear combination of 
the functions $\tilde{\phi}_{-m}$ and $\tilde{\phi}_{i m+1}$; see (5.9). The remainder function $\psi_{\tau}$ cannot be expressed as a linear combination of the orthonormal Laurent polynomials generated by the recursion formulas (2.7)-(2.10); that is, no linear combination both belongs to $\mathcal{L}_{m-1, i m+1}$ and is orthogonal to $\mathcal{L}_{m-1, i m}$. However, for $i=1$ it follows from the structure of $H_{\tau}$ and the formula (7.1) that a normalization $\tilde{\psi}_{\tau}$ of the Laurent polynomial $\psi_{\tau}$ can be computed with the four-term recursion formula

$$
\delta_{\tau} \tilde{\psi}_{\tau}(x)=\left(x-h_{\tau, \tau}\right) \tilde{\phi}_{i m}(x)-h_{\tau-1, \tau} \tilde{\phi}_{-m+1}(x)-h_{\tau-2, \tau} \tilde{\phi}_{i m-1}(x),
$$

where $\delta_{\tau}:=\left\|\psi_{\tau}\right\|$. For $i>1, \tilde{\psi}_{\tau}$ can be determined with the three-term recursion formula

$$
\delta_{\tau} \tilde{\psi}_{\tau}(x)=\left(x-h_{\tau, \tau}\right) \tilde{\phi}_{i m}(x)-h_{\tau-1, \tau} \tilde{\phi}_{i m-1}(x) .
$$

The following example illustrates the structural differences in $H_{\tau}$ for these two cases.

Example 7.1. Entries of the matrices $H_{\tau}$ for $i=1, m=3, \tau=6$, and for $i=2$, $m=2, \tau=6$, that may be non-vanishing are marked by "*":

$$
\begin{aligned}
H_{6}= & {\left[\begin{array}{llllll}
* & * & & & & \\
* & * & * & * & & \\
& * & * & * & & \\
& * & * & * & * & * \\
& & & * & * & * \\
& & & * & * & *
\end{array}\right] \quad \text { for } i=1, } \\
H_{6}= & {\left[\begin{array}{llllll}
* & * & & & & \\
* & * & * & & & \\
& * & * & * & * & \\
& & * & * & * & \\
& & * & * & * & * \\
& & & & * & *
\end{array}\right] \quad \text { for } i=2 . }
\end{aligned}
$$

Observe the three generally non-zero entries in the last row of $H_{\tau}$ for $i=1$. A four-term recursion formula is required to compute $\tilde{\psi}_{\tau}$ in this case.

For all $i \geq 1$, we can compute a Laurent polynomial $\psi_{\tau+1} \in \mathcal{L}_{m-1, i m+2}$ that is orthogonal to $\mathcal{L}_{m-1, i m+1}$ with the three-term recursion formula

$$
\psi_{\tau+1}(x)=(x-\alpha) \tilde{\psi}_{\tau}(x)-\delta_{\tau} \tilde{\phi}_{i m}(x) .
$$

This produces the modified recursion matrix

$$
\hat{H}_{\tau+1}=\left[\begin{array}{cc}
H_{\tau} & \delta_{\tau} \\
\delta_{\tau} & \alpha
\end{array}\right] .
$$

In order to determine a Radau rule with a node $a \leq \lambda_{1}$, we choose the last diagonal entry of $\hat{H}_{\tau+1}$ so that the matrix

$$
\hat{H}_{\tau+1}^{a}=\left[\begin{array}{cc}
H_{\tau} & \delta_{\tau} \\
\delta_{\tau} & \hat{\alpha}
\end{array}\right]
$$

has an eigenvalue at $a$. This is equivalent to requiring that $a$ is a zero of the modified Laurent polynomial

$$
\hat{\psi}(x)=(x-\hat{\alpha}) \tilde{\psi}_{\tau}(x)-\delta_{\tau} \tilde{\phi}_{i m}(x) .
$$


We refer to the requirement $\hat{\psi}(a)=0$ as the Radau condition. It yields

$$
\hat{\alpha}=a-\frac{\delta_{\tau} \tilde{\phi}_{i m}(a)}{\tilde{\psi}_{\tau}(a)} .
$$

We remark that since the eigenvalues of $H_{\tau}$ are the zeros of $\tilde{\psi}_{\tau}$ and they live in the open interval $\left(\lambda_{1}, \lambda_{n}\right)$, the requirement $a \leq \lambda_{1}$ secures that the denominator in (7.3) is non-vanishing. Similarly, we may fix a node larger than or equal to $\lambda_{n}$. This is discussed below.

It is not necessary to compute $\delta_{\tau} \tilde{\phi}_{i m}(a) / \tilde{\psi}_{\tau}(a)$ in order to determine $\hat{\alpha}$. Instead, we proceed similarly as described in [11, 12, 13, 15] for the construction of standard Gauss-Radau rules. Consider the partitioning

$$
x\left[\begin{array}{c}
\Phi_{\tau}(x) \\
\tilde{\psi}_{\tau}(x)
\end{array}\right]=\left[\begin{array}{cc}
H_{\tau} & \delta_{\tau} \\
\delta_{\tau} & \hat{\alpha}
\end{array}\right]\left[\begin{array}{c}
\Phi_{\tau}(x) \\
\tilde{\psi}_{\tau}(x)
\end{array}\right]+\left[\begin{array}{c}
\mathbf{0} \\
\hat{\psi}(x)
\end{array}\right], \quad \mathbf{0} \in \mathbb{R}^{\tau} .
$$

The Radau condition yields the linear system of equations

$$
\left(H_{\tau}-a I\right) \Phi(a)=-\delta_{\tau} \tilde{\psi}_{\tau}(a) \boldsymbol{e}_{\tau},
$$

which after multiplication by $-\delta_{\tau} / \tilde{\psi}_{\tau}(a)$ can be written as

$$
\left(H_{\tau}-a I\right) \hat{\Phi}(a)=\delta_{\tau}^{2} \boldsymbol{e}_{\tau},
$$

where $\hat{\Phi}(a)=-\delta_{\tau} \Phi(a) / \psi_{\tau}(a)$. Upon solution of (7.4), the expression (7.3) reduces to

$$
\hat{\alpha}=a+e_{\tau}^{T} \hat{\Phi}(a) .
$$

It follows from

$$
\hat{\psi}(x)=\delta_{\tau} \psi_{\tau+1}(x)+(\alpha-\hat{\alpha}) \tilde{\psi}_{\tau}(x)
$$

that $\hat{\psi} \in \mathcal{L}_{m-1, i m+2}$ and $\hat{\psi} \perp \mathcal{L}_{m-1, i m}$. Comparing these properties to those of $\psi_{\tau+1}$, we see that by satisfying the Radau condition, we loose one numerator degree of orthogonality.

We also have

$$
\hat{\psi}(x)=(x-a) \omega(x), \quad \omega \in \mathcal{L}_{m-1, i m+1},
$$

where $\omega \perp \mathcal{L}_{m-1, i m}$ with respect to an inner product defined by the positive measure $(x-a) d \mu(x)$. The Laurent polynomial $\omega$ satisfies the conditions of Theorem 5.1 and, thus, has $\tau$ distinct zeros, $x_{1}^{a}, x_{2}^{a}, \ldots, x_{\tau}^{a}$. We may apply Laurent-Lagrange interpolation at these nodes of the function $f \in \mathcal{L}_{2 m-2,2 i m+2}$ to determine the interpolating Laurent-Lagrange polynomial $L_{R}^{a} \in \mathcal{L}_{m-1, i m+1}$ and a remainder term similarly as in Section 5. In this manner, we obtain

$$
f(x)-L_{R}^{a}(x)=\hat{\psi}(x) r(x),
$$

where $r \in \mathcal{L}_{m-1, i m}$. The rational Radau rule

$$
\boldsymbol{e}_{1}^{T} f\left(\hat{H}_{\tau+1}^{a}\right) \boldsymbol{e}_{1},
$$

which also can be written as

$$
\mathcal{G}_{R}^{a}(f)=w_{a} f(a)+\sum_{\nu=1}^{\tau} w_{\nu} f\left(x_{\nu}^{a}\right),
$$

will be exact for all $f \in \mathcal{L}_{2 m-2,2 i m+2}$. If we compare this result with the quadrature rule (4.2) associated with $H_{\tau}$, we see that the rational Radau rule has added one numerator degree of accuracy. 
We also may apply Laurent-Hermite interpolation to produce Hermite interpolating $\hat{L}_{R}^{a}(x) \in \mathcal{L}_{2 m-2,2 i m+2}$, that agrees with $f$ at the $\tau+1$ zeros of $\hat{\psi}$ and that agrees with $f^{\prime}$ at the $\tau$ zeros of $\omega(x)$. Modifying the resulting error term in Theorem 5.4 to reflect the additional node that interpolates $f$ at $a$ and applying Corollary 5.5 yield the following result.

Theorem 7.1. Let $\omega$ be defined by (7.5) with leading coefficient $d$. If $f$ is $2 \tau+1$ times continuously differentiable in the open interval $\left(a, \lambda_{n}\right)$, then

$$
\left(\mathcal{I}-\mathcal{G}_{R}^{a}\right)(f)=\frac{d^{2 \tau+1}}{d t^{2 \tau+1}}\left(t^{2(m-1)} f(t)\right)_{t=c} \frac{\mathcal{I}\left((x-a) \omega^{2}(x)\right)}{d^{2}(2 \kappa+1) !}
$$

for some scalar $c$ in the open interval $\left(a, \lambda_{n}\right)$.

Instead of fixing a node $a \leq \lambda_{1}$, we may define a rational Radau rule with a prescribed node $b \geq \lambda_{n}$. The discussion following Example 7.1 carries over to this situation; we just let $a:=b$ in the formulas. Also an analogue of Theorem 7.1 holds. The only necessary change is that we have to require the function $f$ to be $2 \tau+1$ times continuously differentiable in the open interval $\left(\lambda_{1}, b\right)$. Since $\mathcal{I}\left((x-a) \omega^{2}(x)\right) \geq 0$ and $\mathcal{I}\left((x-b) \omega^{2}(x)\right) \leq 0$, the remainder terms for the rational Radau rules with the prescribed nodes $a \leq \lambda_{1}$ and $b \geq \lambda_{n}$ are of opposite sign if the derivative $\frac{d^{2 \tau+1}}{d t^{2 \tau+1}}\left(t^{2(m-1)} f(t)\right)$ does not change sign in the interval $(a, b)$. This observation yields the following bounds.

Corollary 7.2. Let $f$ be a $2 \tau+1$ times continuously differentiable function in the open interval $(a, b)$. Assume that

$$
\frac{d^{2 \tau+1}}{d x^{2 \tau+1}}\left(x^{2(m-1)} f(x)\right) \geq 0
$$

in this interval. Then

or, equivalently,

$$
\mathcal{G}_{R}^{a}(f) \leq \mathcal{I}(f) \leq \mathcal{G}_{R}^{b}(f)
$$

$$
\boldsymbol{e}_{1}^{T} f\left(\hat{H}_{\tau+1}^{a}\right) \boldsymbol{e}_{1} \leq \boldsymbol{v}^{T} f(A) \boldsymbol{v} \leq \boldsymbol{e}_{1}^{T} f\left(\hat{H}_{\tau+1}^{b}\right) \boldsymbol{e}_{1} .
$$

Bounds analogous to (7.7) can be established if the derivative (7.6) is nonpositive. Sometimes it can be attractive to use a rational Gauss quadrature rule to furnish one of the bounds; cf. Corollary [5.6. This may, for instance, be the case when an upper or lower bound for the spectrum of $A$ is not available.

\section{Numerical EXAMPLES}

The computations in this section are carried out using MATLAB with about 15 significant decimal digits. In all examples, except when explicitly stated otherwise, $A \in \mathbb{R}^{1000 \times 1000}$ and the vector $\boldsymbol{v} \in \mathbb{R}^{1000}$ has normally distributed random entries with mean zero and variance one. The examples, with the exception of Example 8.5, compare the performance of the standard Lanczos method with the rational Lanczos methods for the cases $i=1$ and $i=3$. The size of the examples is chosen small enough to allow the computation of (1.1) in order to be able to evaluate the quadrature error; however, the methods described in this paper can easily be applied to larger problems.

The tables show the errors in the quadrature rules

$$
\boldsymbol{e}_{1}^{T} f\left(\hat{H}_{\tau+1}^{a}\right) \boldsymbol{e}_{1}-\boldsymbol{v}^{T} f(A) \boldsymbol{v} \text { and } \boldsymbol{e}_{1}^{T} f\left(\hat{H}_{\tau+1}^{b}\right) \boldsymbol{e}_{1}-\boldsymbol{v}^{T} f(A) \boldsymbol{v}
$$


between the Radau rule approximations and the functional for several values of $\tau=0 \bmod 4$. This assures that the matrix $H_{\tau}$ defined by (5.8) is of the appropriate dimension for both $i=1$ and $i=3$. We recall that the computation of $\hat{H}_{\tau+1}^{a}$ requires the evaluation of $\tau$ steps of the rational Lanczos process. The columns of the tables are labeled with the quadrature rule used in the differences (8.1) for each case. The table column heading $\boldsymbol{e}_{1}^{T} f\left(\hat{T}^{a}\right) \boldsymbol{e}_{1}$ refers to the standard $(\tau+1)$ point Gauss-Radau rule with a fixed node at $a$. As mentioned in the beginning of Section 7, this rule is exact for all polynomials of degree at most $2 \tau$, and it can be determined with $\tau$ steps of the standard Lanczos process. The $\tau$-point standard Gauss rule (1.5), whose computation also requires $\tau$ steps of the standard Lanczos process, is exact for all polynomials of degree at most $2 \tau-1$, only. The standard Gauss-Radau rule therefore can be expected to yield a smaller integration error than the standard Gauss rule. Section 7 contains analogous results for rational Radau rules. We therefore compare the performance of standard Gauss-Radau and rational Radau rules in this section. All matrix functions $\boldsymbol{v}^{T} f(A) \boldsymbol{v}$ are evaluated by means of the spectral decomposition of the matrix.

For the function $f(x)=\exp (x) / x$, we evaluate (1.1) as $\boldsymbol{v}^{T} \exp (A) A^{-1} \boldsymbol{v}$, where $A^{-1} \boldsymbol{v}$ is computed by solving a linear system of equations. The rational Lanczos process yields approximations

$$
\boldsymbol{e}_{1}^{T} \exp \left(\hat{H}_{\tau+1}^{a}\right) \hat{G}_{\tau+1}^{a} \boldsymbol{e}_{1},
$$

where $a$ is a fixed node smaller than or equal to $\lambda_{1}$. The matrix $\hat{H}_{\tau+1}^{a}$ is defined by (7.2) and the vector $\hat{G}_{\tau+1}^{a} \boldsymbol{e}_{1}$ is the first column of the inverse projection matrix analogous to the projection matrix $G_{\tau}$ of Section 3 , i.e., $\hat{G}_{\tau+1}^{a} e_{1}$ is the first column of the inverse of $\hat{H}_{\tau+1}^{a}$. The computations are analogous for the prescribed node $b \geq \lambda_{n}$.

The standard Lanczos method determines the Lanczos decomposition (1.4) with $m=\tau$, which yields the approximation

$$
\boldsymbol{e}_{1}^{T} \exp \left(\hat{T}_{\tau+1}\right) \hat{T}_{\tau+1}^{-1} \boldsymbol{e}_{1}
$$

This expression is evaluated by first solving a linear system of equations for the vector $\hat{T}_{\tau+1}^{-1} e_{1}$.

TABLE 8.1. Errors in Radau rules with a fixed node at $a=0.3$. The matrix $A$ is symmetric positive definite and Toeplitz, and $f(x)=\exp (-x) / \sqrt{x}$.

\begin{tabular}{cccc}
$\tau$ & $\boldsymbol{e}_{1}^{T} f\left(\hat{T}^{a}\right) \boldsymbol{e}_{1}$ & $\boldsymbol{e}_{1}^{T} f\left(\hat{H}_{i=1}^{a}\right) \boldsymbol{e}_{1}$ & $\boldsymbol{e}_{1}^{T} f\left(\hat{H}_{i=3}^{a}\right) \boldsymbol{e}_{1}$ \\
\hline 4 & $3 \cdot 10^{-2}$ & $8 \cdot 10^{-4}$ & $3 \cdot 10^{-2}$ \\
8 & $1 \cdot 10^{-3}$ & $3 \cdot 10^{-7}$ & $7 \cdot 10^{-6}$ \\
12 & $3 \cdot 10^{-5}$ & $1 \cdot 10^{-10}$ & $3 \cdot 10^{-9}$ \\
16 & $7 \cdot 10^{-7}$ & $2 \cdot 10^{-14}$ & $2 \cdot 10^{-12}$
\end{tabular}

Examples 8.1-8.2. Let $A=\left[a_{j, k}\right]$ be the symmetric positive definite Toeplitz matrix with entries $a_{j, k}=1 /(1+|j-k|)$. Computed results are shown in Tables 8.1 and 8.2 for $f(x)=\exp (-x) / \sqrt{x}$ and in Tables 8.3 and 8.4 for $f(x)=\ln (x)$. We remark that fast direct solution methods are available for linear systems of 
TABLE 8.2. Errors in Radau rules with a fixed node at $b=12.5$. The matrix $A$ is symmetric positive definite and Toeplitz, and $f(x)=\exp (-x) / \sqrt{x}$.

\begin{tabular}{cccc}
$\tau$ & $\boldsymbol{e}_{1}^{T} f\left(\hat{T}^{b}\right) \boldsymbol{e}_{1}$ & $\boldsymbol{e}_{1}^{T} f\left(\hat{H}_{i=1}^{b}\right) \boldsymbol{e}_{1}$ & $\boldsymbol{e}_{1}^{T} f\left(\hat{H}_{i=3}^{b}\right) \boldsymbol{e}_{1}$ \\
\hline 4 & $-1 \cdot 10^{-2}$ & $-5 \cdot 10^{-4}$ & $-1 \cdot 10^{-2}$ \\
8 & $-5 \cdot 10^{-4}$ & $-3 \cdot 10^{-7}$ & $-4 \cdot 10^{-6}$ \\
12 & $-1 \cdot 10^{-5}$ & $-1 \cdot 10^{-10}$ & $-2 \cdot 10^{-9}$ \\
16 & $-3 \cdot 10^{-7}$ & $-2 \cdot 10^{-14}$ & $-2 \cdot 10^{-12}$
\end{tabular}

TABLE 8.3. Errors in Radau rules with a fixed node at $a=0.3$. The matrix $A$ is symmetric positive definite and Toeplitz, and $f(x)=\ln (x)$.

\begin{tabular}{cccc}
$\tau$ & $\boldsymbol{e}_{1}^{T} f\left(\hat{T}^{a}\right) \boldsymbol{e}_{1}$ & $\boldsymbol{e}_{1}^{T} f\left(\hat{H}_{i=1}^{a}\right) \boldsymbol{e}_{1}$ & $\boldsymbol{e}_{1}^{T} f\left(\hat{H}_{i=3}^{a}\right) \boldsymbol{e}_{1}$ \\
\hline 4 & $-2 \cdot 10^{-2}$ & $-8 \cdot 10^{-4}$ & $-2 \cdot 10^{-2}$ \\
8 & $-4 \cdot 10^{-4}$ & $-2 \cdot 10^{-7}$ & $-7 \cdot 10^{-6}$ \\
12 & $-1 \cdot 10^{-5}$ & $-6 \cdot 10^{-11}$ & $-5 \cdot 10^{-9}$ \\
16 & $-3 \cdot 10^{-7}$ & $-1 \cdot 10^{-14}$ & $-3 \cdot 10^{-12}$
\end{tabular}

TABLE 8.4. Errors in Radau rules with a fixed node at $b=12.5$. The matrix $A$ is symmetric positive definite and Toeplitz, and $f(x)=\ln (x)$.

\begin{tabular}{cccc}
$\tau$ & $\boldsymbol{e}_{1}^{T} f\left(\hat{T}^{b}\right) \boldsymbol{e}_{1}$ & $\boldsymbol{e}_{1}^{T} f\left(\hat{H}_{i=1}^{b}\right) \boldsymbol{e}_{1}$ & $\boldsymbol{e}_{1}^{T} f\left(\hat{H}_{i=3}^{b}\right) \boldsymbol{e}_{1}$ \\
\hline 4 & $9 \cdot 10^{-3}$ & $4 \cdot 10^{-4}$ & $9 \cdot 10^{-3}$ \\
8 & $2 \cdot 10^{-4}$ & $1 \cdot 10^{-7}$ & $5 \cdot 10^{-6}$ \\
12 & $6 \cdot 10^{-6}$ & $5 \cdot 10^{-11}$ & $3 \cdot 10^{-9}$ \\
16 & $1 \cdot 10^{-7}$ & $3 \cdot 10^{-14}$ & $2 \cdot 10^{-12}$
\end{tabular}

equations with this kind of matrix; see, e.g., [2, 25. The smallest eigenvalue of $A$ is $\lambda_{1}=0.3863$ and the largest one is $\lambda_{1000}=12.1259$. We chose the Radau parameters $a=0.3$ and $b=12.5$.

TABLE 8.5. Errors in Radau rules with a fixed node at $a=0.05$. The matrix $A$ is a discretization of the differential operator $L$, and $f(x)=\exp (x) / x$.

\begin{tabular}{cccc}
$\tau$ & $\boldsymbol{e}_{1}^{T} f\left(\hat{T}^{a}\right) \boldsymbol{e}_{1}$ & $\boldsymbol{e}_{1}^{T} f\left(\hat{H}_{i=1}^{a}\right) \boldsymbol{e}_{1}$ & $\boldsymbol{e}_{1}^{T} f\left(\hat{H}_{i=3}^{a}\right) \boldsymbol{e}_{1}$ \\
\hline 8 & $3 \cdot 10^{0}$ & $-8 \cdot 10^{-5}$ & $-1 \cdot 10^{-5}$ \\
12 & $2 \cdot 10^{0}$ & $-1 \cdot 10^{-6}$ & $-3 \cdot 10^{-8}$ \\
16 & $2 \cdot 10^{0}$ & $-4 \cdot 10^{-9}$ & $-4 \cdot 10^{-11}$
\end{tabular}

Examples 8.3-8.4. The matrix $A$ in this example is obtained by discretizing the self-adjoint differential operator $L(u)=\frac{1}{10} u_{x x}-100 u_{y y}$ in the unit square. Each derivative is approximated by the standard three-point stencil with 40 equally 
TABLE 8.6. Errors in Radau rules with a fixed node at $b=45$. The matrix $A$ is a discretization of the differential operator $L$, and $f(x)=\exp (x) / x$.

\begin{tabular}{cccc}
$\tau$ & $\boldsymbol{e}_{1}^{T} f\left(\hat{T}^{b}\right) \boldsymbol{e}_{1}$ & $\boldsymbol{e}_{1}^{T} f\left(\hat{H}_{i=1}^{b}\right) \boldsymbol{e}_{1}$ & $\boldsymbol{e}_{1}^{T} f\left(\hat{H}_{i=3}^{b}\right) \boldsymbol{e}_{1}$ \\
\hline 8 & $-3 \cdot 10^{0}$ & $9 \cdot 10^{-5}$ & $1 \cdot 10^{-5}$ \\
12 & $-1 \cdot 10^{0}$ & $9 \cdot 10^{-7}$ & $3 \cdot 10^{-8}$ \\
16 & $-4 \cdot 10^{-1}$ & $3 \cdot 10^{-9}$ & $3 \cdot 10^{-11}$
\end{tabular}

TABLE 8.7. Errors in Radau rules with a fixed node at $a=0.05$. The matrix $A$ is a discretization of the differential operator $L$, and $f(x)=x^{-1 / 2}$.

\begin{tabular}{cccc}
$\tau$ & $\boldsymbol{e}_{1}^{T} f\left(\hat{T}^{a}\right) \boldsymbol{e}_{1}$ & $\boldsymbol{e}_{1}^{T} f\left(\hat{H}_{i=1}^{a}\right) \boldsymbol{e}_{1}$ & $\boldsymbol{e}_{1}^{T} f\left(\hat{H}_{i=3}^{a}\right) \boldsymbol{e}_{1}$ \\
\hline 8 & $4 \cdot 10^{-1}$ & $2 \cdot 10^{-4}$ & $-1 \cdot 10^{-5}$ \\
12 & $2 \cdot 10^{-1}$ & $1 \cdot 10^{-6}$ & $-3 \cdot 10^{-8}$ \\
16 & $1 \cdot 10^{-1}$ & $1 \cdot 10^{-9}$ & $-4 \cdot 10^{-11}$
\end{tabular}

TABLE 8.8. Errors in Radau rules with a fixed node at $b=45$. The matrix $A$ is a discretization of the differential operator $L$, and $f(x)=x^{-1 / 2}$.

\begin{tabular}{cccc}
$\tau$ & $\boldsymbol{e}_{1}^{T} f\left(\hat{T}^{b}\right) \boldsymbol{e}_{1}$ & $\boldsymbol{e}_{1}^{T} f\left(\hat{H}_{i=1}^{b}\right) \boldsymbol{e}_{1}$ & $\boldsymbol{e}_{1}^{T} f\left(\hat{H}_{i=3}^{b}\right) \boldsymbol{e}_{1}$ \\
\hline 8 & $-3 \cdot 10^{-1}$ & $-1 \cdot 10^{-4}$ & $1 \cdot 10^{-5}$ \\
12 & $-1 \cdot 10^{-1}$ & $-4 \cdot 10^{-7}$ & $3 \cdot 10^{-8}$ \\
16 & $-4 \cdot 10^{-2}$ & $-4 \cdot 10^{-10}$ & $3 \cdot 10^{-11}$
\end{tabular}

spaced interior nodes in each space dimension. Homogeneous boundary conditions are used. This yields a $1600 \times 1600$ symmetric positive definite matrix $A$. The initial vector $\boldsymbol{v}$ for the standard and rational Lanczos processes is chosen to be the unit vector with all entries $1 / \sqrt{1600}$. Tables 8.5 and 8.6 display the differences between the Radau approximations and the functional for the standard Lanczos method and rational Lanczos methods for the functions $f(x)=\exp (x) / x$. Analogous results for the function $f(x)=1 / \sqrt{x}$ are shown by the Tables 8.7 and 8.8. The smallest eigenvalue of $A$ is $\lambda_{1}=0.0646$ and the largest eigenvalue is $\lambda_{1600}=43.9354$. We chose the Radau parameters $a=0.05$ and $b=45$.

Example 8.5. Let $A$ be the symmetric positive definite tridiagonal Toeplitz matrix $[-1,2,-1]$ of order 1000 and $f(x)=\ln (x)$. The extreme eigenvalues of $A$ are $\lambda_{1}=0.00001$ and $\lambda_{1000}=3.99999$. We chose the Radau parameters $a=9 \cdot 10^{-6}$ and $b=4.5$. The spectrum of $A$ is close to zero and convergence of the approximation methods is slow for the cases $i=1,2,3$, though superior to the convergence achieved with the Lanczos method. Figure 8.1 illustrates the convergence of $\boldsymbol{e}^{T} f\left(\hat{H}^{a}\right) \boldsymbol{e}$ and $\boldsymbol{e}^{T} f\left(\hat{H}^{b}\right) \boldsymbol{e}$ to $\boldsymbol{v}^{T} f(A) \boldsymbol{v}$ from above and below, respectively. The plots illustrate the case $i=2$ for $\tau=3,6,9,12,15,18$ and the case $i=3$ for $\tau=4,8,12,16,20$. 


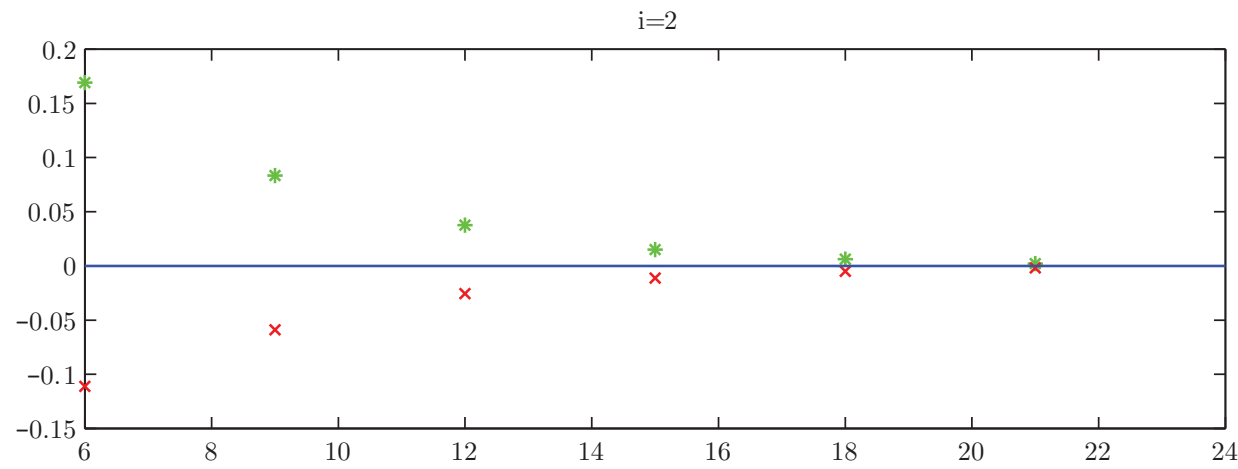

$\mathrm{i}=3$

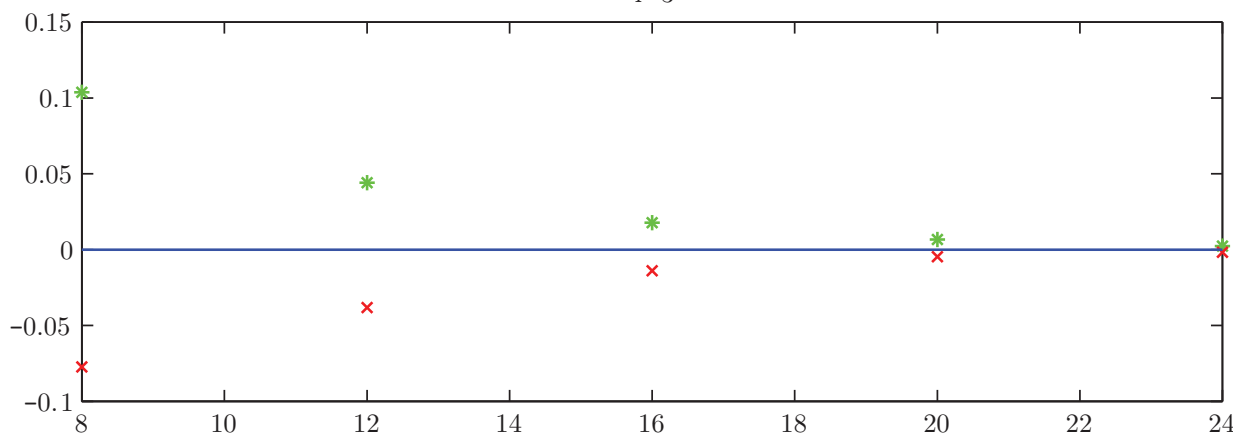

Figure 8.1. Convergence of the error in $\boldsymbol{e}^{T} \hat{H}^{a} \boldsymbol{e}$ and $\boldsymbol{e}^{T} \hat{H}^{b} \boldsymbol{e}$ to zero for $i=2$ and $i=3$ for increasing values of $\tau$. The quadrature values $\boldsymbol{e}^{T} \hat{H}^{a} \boldsymbol{e}$ and $\boldsymbol{e}^{T} \hat{H}^{b} \boldsymbol{e}$ are denoted by $(*)$ and $(\times)$, respectively.

\section{Conclusion}

Standard Gauss and Gauss-Radau rules are associated with symmetric tridiagonal matrices. This paper explores the structure of the analogous matrices for rational Gauss and Gauss-Radau rules. Properties of these quadrature rules are investigated. Applications to the computation of inexpensive upper and lower bounds for certain matrix functionals are described and illustrated.

\section{ACKNOWLEDGMENT}

We would like to thank the referees for comments that improved the presentation.

\section{REFERENCES}

[1] E. J. Allen, J. Baglama, and S. K. Boyd, Numerical approximation of the product of the square root of a matrix with a vector, Linear Algebra Appl., 310 (2000), pp. 167-181. MR.1753175(2001c:65050)

[2] G. S. Ammar and W. B. Gragg, Superfast solution of real positive definite Toeplitz systems, SIAM J. Matrix Anal. Appl., 9 (1988), pp. 61-76. MR938136(89b:65065)

[3] Z. Bai, M. Fahey, and G. H. Golub, Some large scale matrix computation problems, J. Comput. Appl. Math., 74 (1996), pp. 71-89. MR1430368(97k:65073) 
[4] B. Beckermann and L. Reichel, Error estimation and evaluation of matrix functions via the Faber transform, SIAM J. Numer. Anal., 47 (2009), pp. 3849-3883. MR 2576523 (2010m:30047)

[5] M. Benzi and P. Boito, Quadrature rule based bounds for functions of adjacency matrices, Linear Algebra Appl., 433 (2010), pp. 637-652. MR2653828(2011d:65106)

[6] C. F. Borges and W. B. Gragg, A parallel divide and conquer algorithm for the generalized real symmetric definite tridiagonal eigenproblem, in Numerical Linear Algebra, eds. L. Reichel, A. Ruttan, and R. S. Varga, de Gruyter, Berlin, 1993, pp. 11-29. MR1244151 (94k:65051)

[7] D. Calvetti and L. Reichel, Lanczos-based exponential filtering for discrete ill-posed problems, Numer. Algorithms, 29 (2002), pp. 45-65. MR1896945 (2003e:65063)

[8] C. Díaz-Mendoza, P. Gonzáles-Vera, M. Jiménez Paiz, and O. Njåstad, Orthogonality and recurrence for ordered Laurent polynomial sequences, J. Comput. Appl. Math., 235 (2010), pp. 982-997. MR2727633(2011m:30005)

[9] V. Druskin and L. Knizhnerman, Extended Krylov subspace approximations of the matrix square root and related functions, SIAM J. Matrix Anal. Appl., 19 (1998), pp. 755-771. MR:1616584 (99f:65063)

[10] V. Druskin, L. Knizhnerman, and M. Zaslavsky, Solution of large scale evolutionary problems using rational Krylov subspaces with optimized shifts, SIAM J. Sci. Comput., 31 (2009), pp. 3760-3780. MR 2556561 (2011d:30067)

[11] W. Gautschi, The interplay between classical analysis and (numerical) linear algebra - a tribute to Gene H. Golub, Electron. Trans. Numer. Anal., 13 (2002), pp. 119-147. MR1961202 (2004b:65034)

[12] W. Gautschi, Orthogonal Polynomials: Computation and Approximation, Oxford University Press, Oxford, 2004. MR 2061539(2005e:42001)

[13] G. H. Golub, Some modified matrix eigenvalue problems, SIAM Rev., 15 (1973), pp. 318-337. MR 0329227(48:7569)

[14] G. H. Golub and G. Meurant, Matrices, moments and quadrature, in Numerical Analysis 1993, eds. D. F. Griffiths and G. A. Watson, Longman, Essex, 1994, pp. 105-156. MR.1267758 (95b:65042)

[15] G. H. Golub and G. Meurant, Matrices, Moments and Quadrature with Applications, Princeton University Press, Princeton, 2010. MR2582949 (2011a:65001)

[16] G. H. Golub and J. H. Welsch, Calculation of Gauss quadrature rules, Math. Comp., 23 (1969), pp. 221-230. MR 0245201(39:6513)

[17] A. A. Gonchar and G. López Lagomasino, On Markov's theorem for multipoint Padé approximants, Math. USSR Sb., 34 (1978), pp. 449-459.

[18] M. Gu and S. C. Eisenstat, A divide-and-conquer algorithm for the symmetric tridiagonal eigenproblem, SIAM J. Matrix Anal. Appl., 16 (1995), pp. 172-191. MR1311425 (95j:65035)

[19] N. J. Higham, Functions of Matrices: Theory and Computation, SIAM, Philadelphia, 2008. MR2396439(2009b:15001)

[20] C. Jagels and L. Reichel, The extended Krylov subspace method and orthogonal Laurent polynomials, Linear Algebra Appl., 431 (2009), pp. 441-458. MR2528942 (2010j:65060)

[21] C. Jagels and L. Reichel, Recursion relations for the extended Krylov subspace method, Linear Algebra Appl., 434 (2011), pp. 1716-1732. MR2775750(2012a:65114)

[22] W. B. Jones and O. Njåstad, Orthogonal Laurent polynomials and strong moment theory: a survey, J. Comput. Appl. Math., 105 (1999), pp. 51-91. MR1690578(2000d:30054)

[23] G. López Lagomasino, L. Reichel, and L. Wunderlich, Matrices, moments, and rational quadrature, Linear Algebra Appl., 429 (2008), pp. 2540-2554. MR2456794 (2009h:65035)

[24] O. Njåstad and W. J. Thron, The theory of sequences of orthogonal L-polynomials, in Padé Approximants and Continued Fractions, eds. H. Waadeland and H. Wallin, Det Kongelige Norske Videnskabers Selskab, Skrifter 1, 1983, pp. 54-91.

[25] R. Vandebril, M. Van Barel, and N. Mastronardi, Matrix Computations and Semiseparable Matrices, Vol. 1: Linear Systems, Johns Hopkins University Press, Baltimore, 2007. MR2378139(2009d:15002) 
Department of Mathematics and Computer Science, Hanover College, Hanover, InDIANA 47243

E-mail address: jagels@hanover.edu

Department of Mathematical Sciences, Kent State University, Kent, Ohio 44242

E-mail address: reichel@math.kent.edu 